\title{
Comprehensive transcriptome and methylome analysis delineates the biological basis of hair follicle development and wool-related traits in Merino sheep
}

Bingru Zhao', Hanpeng Luo ${ }^{1}$, Junmin $\mathrm{He}^{2}$, Xixia Huang ${ }^{3}$, Siqian Chen ${ }^{1}$, Xuefeng Fu², Weidan Zeng ${ }^{3}$, Yuezhen Tian², Shuli Liu', Cong-jun Li ${ }^{4}$, George E. Liu', Lingzhao Fang ${ }^{5^{*}} \mathbb{D}$, Shengli Zhang ${ }^{1 *}$ and Kechuan Tian ${ }^{6^{*}}$

\begin{abstract}
Background: Characterization of the molecular mechanisms underlying hair follicle development is of paramount importance in the genetic improvement of wool-related traits in sheep and skin-related traits in humans. The Merino is the most important breed of fine-wooled sheep in the world. In this study, we systematically investigated the complexity of sheep hair follicle development by integrating transcriptome and methylome datasets from Merino sheep skin.

Results: We analysed 72 sequence datasets, including DNA methylome and the whole transcriptome of four gene types, i.e. protein-coding genes (PCGs), IncRNAs, circRNAs, and miRNAs, across four embryonic days (E65, E85, E105, and E135) and two postnatal days (P7 and P30) from the skin tissue of 18 Merino sheep. We revealed distinct expression profiles of these four gene types across six hair follicle developmental stages, and demonstrated their complex interactions with DNA methylation. PCGs with stage-specific expression or regulated by stage-specific IncRNAs, circRNAs, and miRNAs were significantly enriched in epithelial differentiation and hair follicle morphogenesis. Regulatory network and gene co-expression analyses identified key transcripts controlling hair follicle development. We further predicted transcriptional factors (e.g. KLF4, LEF1, HOXC13, RBPJ, VDR, RARA, and STAT3) with stage-specific involvement in hair follicle morphogenesis. Through integrating these stage-specific genomic features with results from genome-wide association studies (GWAS) of five wool-related traits in 7135 Merino sheep, we detected developmental stages and genes that were relevant with wool-related traits in sheep. For instance, genes that were specifically upregulated at E105 were significantly associated with most of woolrelated traits. A phenome-wide association study (PheWAS) demonstrated that candidate genes of wool-related
\end{abstract}

\footnotetext{
*Correspondence: Lingzhao.fang@igmm.ed.ac.uk; zhangslcau@cau.edu.cn; tiankechuan@163.com

${ }^{5}$ MRC Human Genetics Unit at the Institute of Genetics and Cancer, University of Edinburgh, Edinburgh, UK

${ }^{1}$ National Engineering Laboratory for Animal Breeding, Key Laboratory of Animal Genetics, Breeding, and Reproduction, Ministry of Agriculture, College of Animal Science and Technology, China Agricultural University, Beijing, China

${ }^{6}$ Institute of Animal Science and Veterinary Medicine, Shandong Academy of Agricultural Sciences, Jinan, China

Full list of author information is available at the end of the article
}

(C) The Author(s). 2021 Open Access This article is licensed under a Creative Commons Attribution 4.0 International License, which permits use, sharing, adaptation, distribution and reproduction in any medium or format, as long as you give appropriate credit to the original author(s) and the source, provide a link to the Creative Commons licence, and indicate if changes were made. The images or other third party material in this article are included in the article's Creative Commons licence, unless indicated otherwise in a credit line to the material. If material is not included in the article's Creative Commons licence and your intended use is not permitted by statutory regulation or exceeds the permitted use, you will need to obtain permission directly from the copyright holder. To view a copy of this licence, visit http://creativecommons.org/licenses/by/4.0/. The Creative Commons Public Domain Dedication waiver (http://creativecommons.org/publicdomain/zero/1.0/) applies to the data made available in this article, unless otherwise stated in a credit line to the data. 
traits (e.g. SPHK1, GHR, PPP1R27, CSRP2, EEF1A2, and PTPN1) in sheep were also significantly associated with dermatological, metabolic, and immune traits in humans.

Conclusions: Our study provides novel insights into the molecular basis of hair follicle morphogenesis and will serve as a foundation to improve breeding for wool traits in sheep. It also indicates the importance of studying gene expression in the normal development of organs in understanding the genetic architecture of economically important traits in livestock. The datasets generated here are useful resources for functionally annotating the sheep genome, and for elucidating early skin development in mammals, including humans.

Keywords: Developmental stage, DNA methylation, Genome-wide association study, Hair follicle morphogenesis, Sheep, Transcriptome

\section{Background}

In mammals, hair follicles are crucial for temperature regulation, physical protection, sweat and sebum dispersion, sensory and tactile functions, and social interactions $[1,2]$. The hair follicle morphogenesis in sheep is often divided into five stages, including induction (embryonic day 65, E65), organogenesis (E85, primary and secondary follicles related), differentiation (E105 E135, especially for secondary follicles), maturation (postnatal day 7, P7), and postnatal hair cycle stages (P30) $[3,4]$. The number of dermal papilla cells and the size of hair placode are associated with the diameter, crimp, and density of wool fibres [3], which are of high economic value in sheep industry. In addition, the normal development of hair follicle highly interacts with the immune system, such as the immune capacity of hair follicle [57]. The protection of hair follicles and their stem cells against the autoimmune system is fundamental to guarantee the normal development of hair follicles $[3,8]$. Similar to head hair follicles in humans, the occurrence and growth of hair follicles in sheep are independent from neighbouring follicles. The sweat glands and wool follicles as unique features of adult sheep trunk skin resemble those in human axillary skin [9]. Therefore, the characterization of molecular mechanisms underlying the hair follicle morphogenesis in sheep will help understand the genetic basis of wool-related traits and can serve as a valuable model for skin-relevant diseases in humans.

The normal morphogenesis and development of hair follicle is the culmination of spatiotemporal expression of genes under the control of genetic and epigenetic elements, such as long non-coding RNA (lncRNA), microRNAs (miRNAs), circular RNAs (circRNAs), and DNA methylation $[10,11]$. Although several studies have investigated the development of skin in sheep previously, they were limited in terms of developmental stages and gene types. For instance, Nie et al. [4] explored the global changes of lncRNAs and mRNAs at two developmental stages during the induction of primary wool follicles in Carpet sheep. Zhao et al. [12] investigated the involvement of lncRNA-miRNA-mRNA interaction networks in the hair follicle induction across three developmental stages in Aohan sheep.

The majority of genetic variants discovered in genome-wide association studies (GWAS) are noncoding [13]. Better characterization of the regulatory elements in the livestock genome, such as through the efforts of the ongoing Functional Annotation of Animal Genomes (FAANG) and the Farm animal GenotypeTissue Expression (FarmGTEx) projects [14-16], is therefore essential for biologically interpreting the GWAS loci of complex traits of economic value. Furthermore, integrating functional annotations that are specific to tissues and developmental stages with GWAS results can help reveal the causal tissues, developmental stages, and genes for complex traits and diseases [1719].

In this study (Additional file 1: Fig. S1), to comprehensively characterize the molecular mechanisms underpinning hair follicle development in sheep, we sequenced DNA methylation and the whole transcriptome of four gene types, including protein-coding genes (PCGs), miRNAs, circRNAs, and lncRNAs, across six important hair follicle developmental stages (i.e. E65, E85, E105, E135, P7, and P30) in the skin tissue of 18 Merino sheep. In total, we newly generated 72 sequence datasets, which enabled us to identify the key genes, transcriptional factors (TFs), signalling pathways, and interaction networks regulating hair follicle morphogenesis across developmental stages. We then integrated these multi-molecular features specific to each developmental stage with GWAS signals of five wool-related traits and one growth trait to understand the genetic and biological basis of such traits in sheep. These traits included mean fibre diameter (MFD), coefficient of variation of the fibre diameter (CVFD), crimp number (CN), mean staple length (MSL), greasy fleece weight (GFW), and live weight (LW) in 7135 Merino sheep. The resources and findings generated here will provide an opportunity to better annotate the sheep genome by predicting novel non-coding genes and inferring the function of 
unannotated genes via co-expression networks, as well as to enhance the genetic improvement of wool traits in sheep.

\section{Results \\ Histological changes of hair follicles across developmental stages}

We performed the haematoxylin-eosin (H\&E) staining for horizontal and longitudinal sections of skin to observe the histological changes of hair follicles across six developmental stages (i.e. E65, E85, E105, E135, P7, and P30) (Fig. 1a, b). The hair placode (Pc) and dermal condensate (DC) started to form at E65, indicating the induction of hair follicles. At E85, the number of primary follicles (PFs) increased and the secondary follicles (SFs) started to form. At E105, the SFs started to differentiate, and the number of secondary-derived follicles increased at E135. At P7, hair follicles matured with a complete structure, and hair shafts emerged through the epidermis. At P30, hair follicles entered into the anagen phase, during which the root of the hair divides rapidly, adding to the hair shaft. The numbers of PF and SF, the SF/PF ratios (an indicator of wool fineness), and the body weights and lengths through all these six stages are shown in Fig. 1c. In general, the numbers of PF and SF peaked at E85 and E105, respectively, and as expected, body weights (BW) and body lengths (BL) increased across developmental stages.

\section{Identification of stage-specific transcripts and DNA} methylation regions during hair follicle development In total, we generated 72 distinct RNA sequencing datasets for PCGs, lncRNAs, circRNAs, and miRNAs across six developmental stages in 18 animals, producing $3,998,197,856$ clean reads with an average mapping rate of $90.46 \%$. We also generated 18 methylated DNA immunoprecipitation sequencing (MeDlP-Seq) datasets, yielding 1,028,125,898 clean reads with an average mapping rate of $97.19 \%$. The mapping details of all the 72 datasets are described in Additional file 2: Table S1.

We quantified and normalized the expression levels of four gene types across samples, including 19,229 PCGs, 10,193 lncRNAs (1540 existing and 8653 novel), 151 known miRNAs $(98.7 \%$ existing in the miRBase database (Release 22.1) [20]), and 41,369 novel circRNAs (Additional file 3: Table S2). We identified a total of $1,730,765$ methylation peaks in the 18 samples, and the number of peaks across major genomic features (e.g. $5^{\prime}$ UTR, exon and 3'UTR) in each sample is summarized in Additional file 4: Table S3.

The principal component analysis (PCA) of all 18 samples based on the five molecular profiles consistently revealed that the developmental stage was the major factor distinguishing samples (Fig. 2a, Additional file 5: Fig. S2). Distributions of the expression levels of four gene types and DNA methylation levels across developmental stages are shown in Fig. 2b. Overall, the majority of

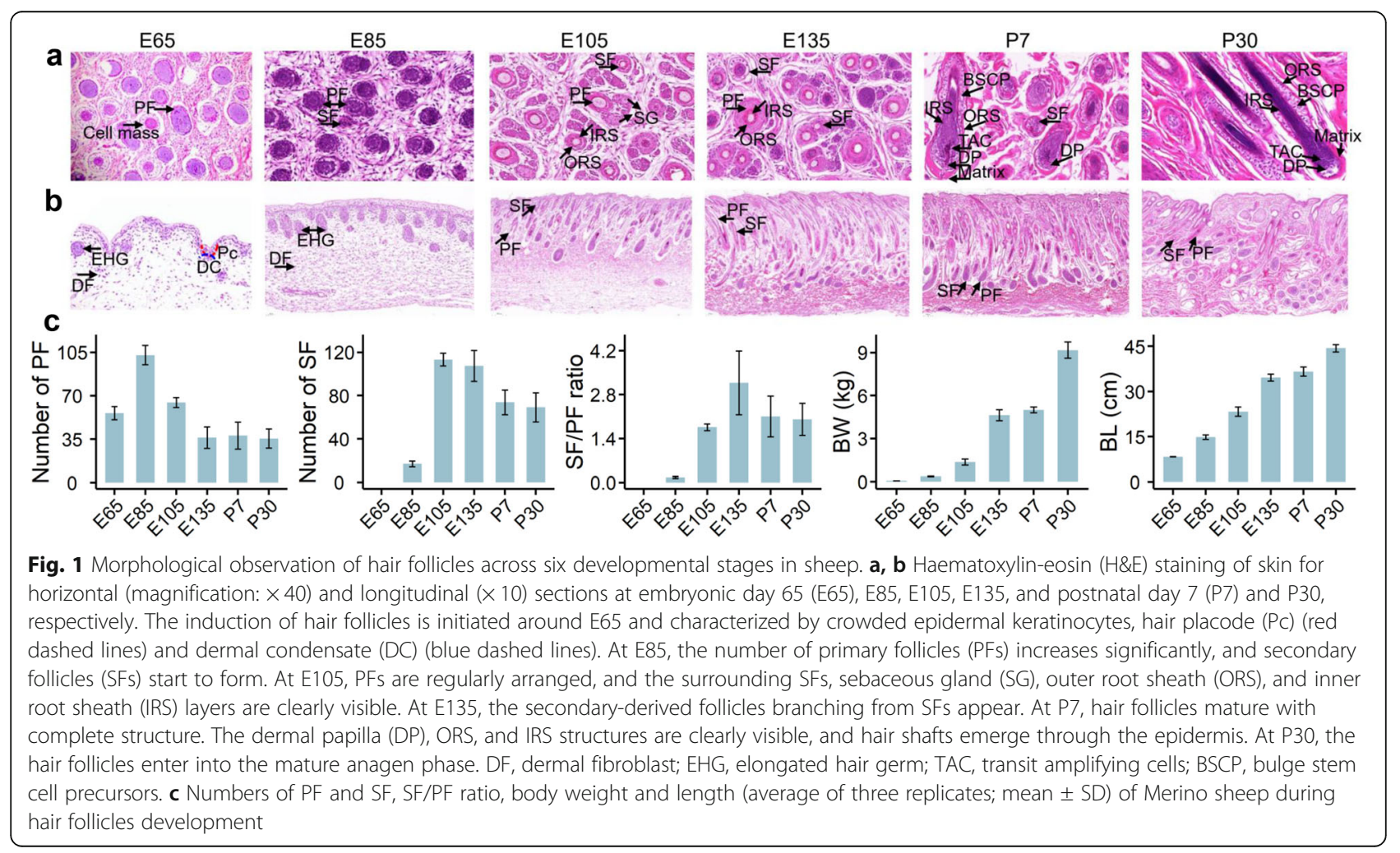




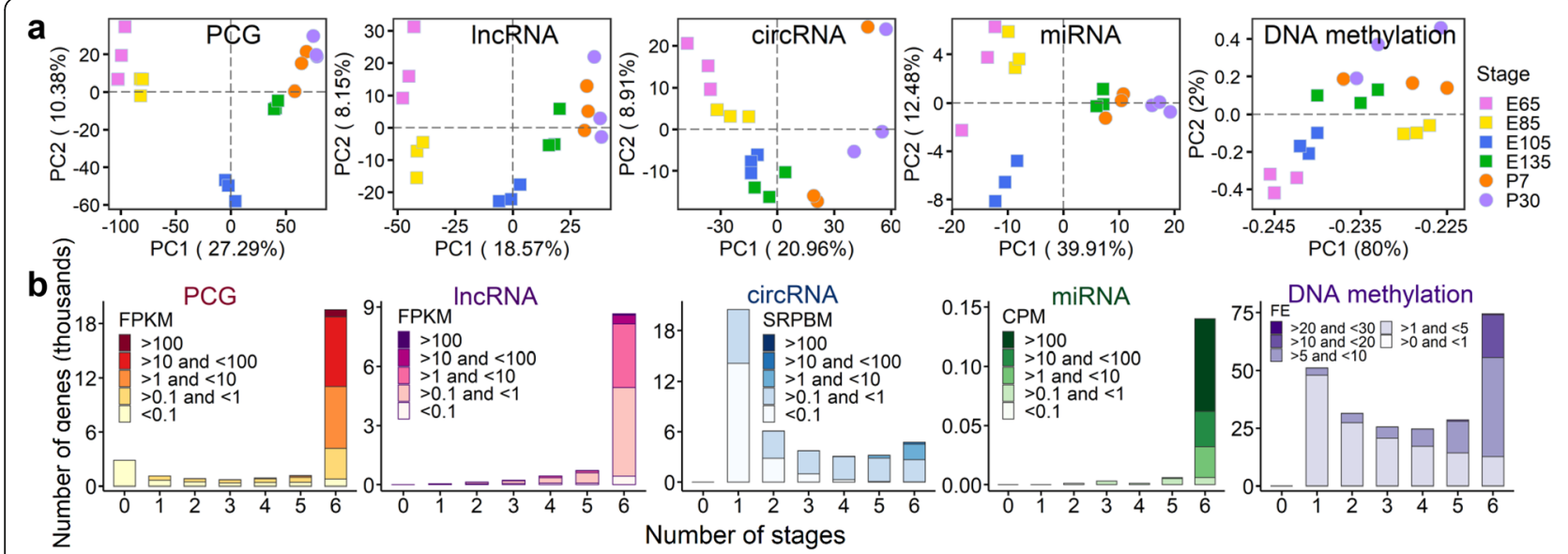

Fig. 2 General characteristics of molecular features in sheep skin tissue across developmental stages. a Principal component analysis (PCA) of all 18 samples based on the expression levels of four gene types and DNA methylation. PCG, protein-coding gene. $\mathbf{b}$ The distribution of gene expression and DNA methylation across number of stages. FPKM, fragments per kilobase of exon model per million mapped fragments; SRPBM, spliced reads per billion mapping; CPM, counts per million; FE, fold enrichment

PCGs, lncRNAs, and miRNAs were ubiquitously expressed across all six stages, while circRNAs showed clear stage-specific expression. We found that all circRNAs were derived from 6477 parental genes, with the majority of $(27,233$ out of 41,369$)$ them from coding DNA sequences (CDS) (Additional file 6: Fig. S3a). Of these parental genes, 4550 produced more than one circRNAs (Additional file 6: Fig. S3b), suggesting that circRNAs are abundant during skin development. Furthermore, we explored the stage specificity of PCGs, IncRNAs, circRNAs, miRNAs, and methylated regions (MRs) during hair follicle morphogenesis. The greatest number of stage-specific transcripts was detected at E65 (the induction of hair follicle) and at P30 (the anagen of hair follicle) (Additional file 7: Fig. S4), which was in line with the development of hair follicles (Fig. 1a-c).

\section{Dynamic expression patterns of PCGs during the hair follicle morphogenesis}

The gene set enrichment analysis (GSEA) of stagespecific PCGs revealed significantly (FDR <0.05) enriched Gene Ontology (GO) terms, which were distinct across developmental stages (Fig. 3a, b, Additional file 8: Table S4). For instance, the PCGs upregulated at E65, E85, E105, E135, P7, and P30 were significantly enriched in chromosome assembly, neurological system, muscle development, regulation of keratinocyte differentiation, keratinocyte differentiation, and lipid metabolism, respectively. The upregulated PCGs at E135-P30 were also significantly enriched in various immune processes such as lymphocyte polarity establishment and $\mathrm{T}$ cell-mediated cytotoxicity (Fig. 3a). The downregulated PCGs at E105 were significantly enriched in muscle development and sensory perception of stimuli, while the downregulated PCGs at E135-P30 were significantly enriched in the keratinocyte and epidermal cell differentiation, skin development, and immune processes (Fig. 3b).

To investigate whether PCGs with stage-specific expression were collectively regulated by certain TFs, we performed a motif enrichment analysis on the promoters (1500 bp up- and $500 \mathrm{bp}$ downstream of transcription start sites, TSS) of upregulated and downregulated stage-specific PCGs separately. We detected 29 and 27 significant motifs (FDR <0.05) for upregulated and downregulated PCGs, respectively, mainly including the $\operatorname{KLF}(n=33)$, EGR $(n=32)$, and SOX $(n=17)$ motif families (Additional file 9: Table S5). We found that the enrichment of motifs and the expression of their target TFs were stage-specific, indicating that these TFs might play vital roles during embryo and hair follicle development (Fig. 3c, d). For instance, PLAG1, EGR1, EGR3, GATA6, and TFAP2C are essential for embryonic organ formation [21-23], while KLF4, LEF1, HOXC13, RBPJ, VDR, RARA, and STAT3 are crucial for hair follicle differentiation and development [24-30]. We further found that NFKB1, NFKB2, and IRF1, which participate in the immune function and inflammation [31], were significant at E105 and E135.

We grouped all stage-specific PCGs into six clusters with different expression trends across stages, and also found that these clusters exhibited distinct biological functions and motif enrichment patterns (Fig. 3e). For instance, PCGs in Cluster1, whose expression levels gradually increased across developmental stages, were significantly $(P<0.01)$ enriched in epidermal cell differentiation and lipid metabolism. The promoters of these PCGs were also significantly enriched for early growth- 


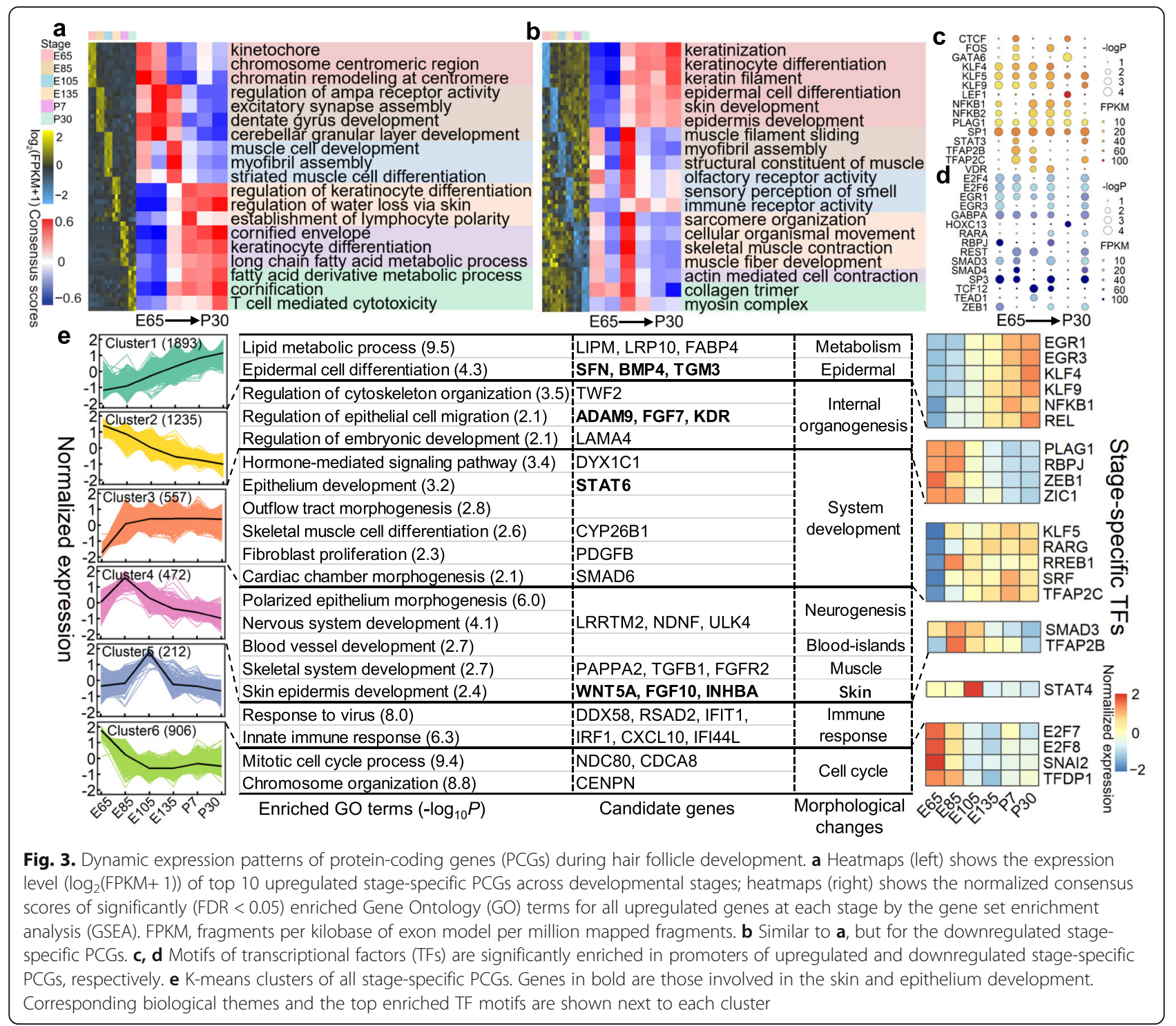

specific TF motifs, such as EGR3 [32] and KLF4 [33]. In contrast to Cluster1, we observed that expression levels of PCGs in Cluster2 gradually decreased across developmental stages. These genes were significantly enriched in internal organogenesis, and their promoters were significantly enriched for organogenesis-related TFs (e.g. ZEB1) [34] and notch signalling pathway-related TFs (e.g. RBPJ) [27]. The PCGs in Cluster5 showed the highest expression levels at E105 and were significantly $(P<$ 0.01 ) enriched in the immune response. Their promoters were significantly enriched for motifs of STAT4, which plays a key role in the immune system [35]. The expression patterns of enriched TFs were consistent with the enrichment of their motifs across developmental stages (Fig. 3e), providing more evidence that these TFs paly crucial regulatory roles in gene expression during normal skin development.

\section{Stage-specific regulatory mechanisms of non-coding RNAs and DNA methylation}

To investigate the roles of circRNAs in the gene regulation during hair follicle development, we performed a GO enrichment analysis for parental genes of stagespecific circRNAs. The parental genes of upregulated circRNAs participated in embryo development at E65, skin and cellular development at E85, system development and epithelial cell proliferation at E105, skin development at E135, metabolic process at P7, and immune response at P30 (Fig. 4a). The parental genes of downregulated circRNAs were significantly $(P<0.01)$ enriched in skin development at E65, protein catabolism at E85, immune response at E105, and central nervous system (CNS) at E135 to P30 (Additional file 10: Fig. S5). We further divided the stage-specific circRNAs into six clusters according to their expression trends across 


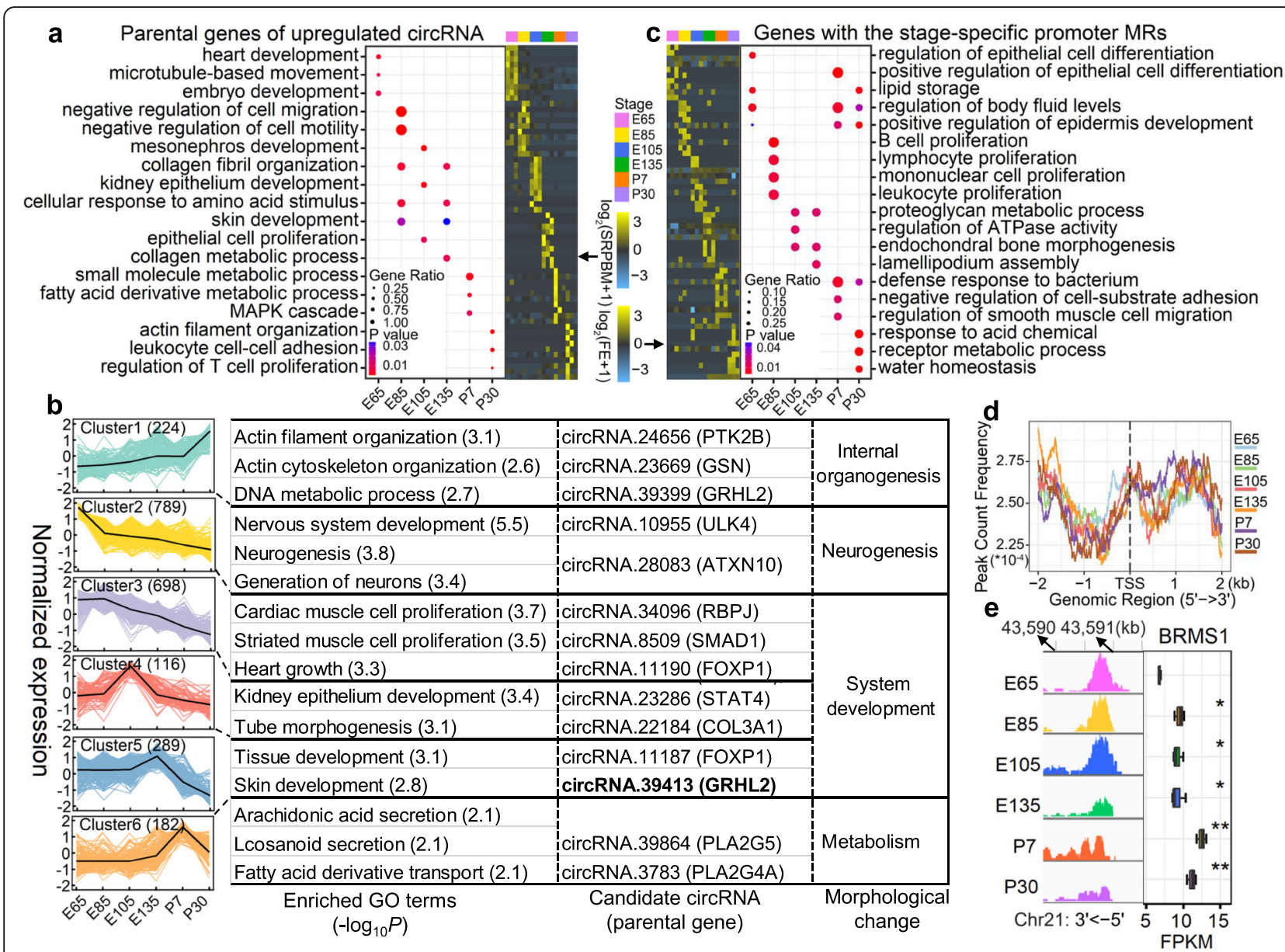

Fig. 4 Stage-specific regulatory mechanisms of circRNAs and DNA methylation. a Heatmap shows the expression $\left(\log _{2}\left(S_{R P B M+1)}\right)\right.$ of upregulated stage-specific circRNAs across developmental stages, and the bubble plot shows the top three enriched gene ontology (GO) terms for parental genes of the upregulated circRNAs across stages. b K-means clustering of all stage-specific circRNAs. Corresponding biological processes are shown next to each cluster. $\mathbf{c}$ Heatmap shows the DNA methylation signals $\left(\log _{2}(\mathrm{FE}+1)\right)$ of stage-specific methylated regions (MRs) in promoters (1500 bp upstream and 500 bp downstream of transcriptional start sites, TSSs). FE, fold enrichment. The bubble plot shows the top five enriched gene ontology (GO) terms for genes with stage-specific MRs in promoter. $\mathbf{d}$ Distribution of DNA methylation levels around TSSs across all six skin developmental stages. e The methylation level of BRMS1 promoter decreases, whereas its expression level increases across developmental stages

the developmental stages (Fig. 4b). The expression levels of 224 circRNAs in Cluster1 increased across developmental stages, whose parental genes were significantly enriched in internal organogenesis. Conversely, cluster2 comprised of 789 circRNAs with developmentally decreased expression levels, whose parental genes were significantly enriched in neurogenesis. Meanwhile, the 289 circRNAs in cluster 5 demonstrated the highest expression levels at E135, whose parental genes were significantly enriched in skin development, such as circRNA.39413 (GRHL2) (Fig. 4b).

Furthermore, we explored the biological functions of genes that were regulated by lncRNAs and miRNAs across developmental stages. For instance, targets of both upregulated and downregulated lncRNAs were significantly enriched in immune and metabolic processes
(Additional file 11: Fig. S6a, b). The targets of upregulated stage-specific miRNAs were significantly enriched in homeostasis-related at E65, internal organogenesis at E85, cellular developmental process at E105, and hormone secretion-related at P30 (Additional file 11: Fig. S6c). The targets of downregulated stage-specific miRNAs were significantly enriched in the hormone secretion at E85, metabolic process at P30, and notch signalling pathway at the rest of stages (Additional file 11: Fig. S6d). Overall, these results indicated that noncoding RNAs play important roles in skin development by distinctly regulating the expression of their target genes across developmental stages.

The functional enrichment analysis revealed that genes with the stage-specific promoter MRs were significantly enriched in epithelial cell differentiation at E65 and P7, 
and in immune processes at E85 (Fig. 4c). In general, we observed that the upstream DNA methylation levels of TSSs were lower at the later stages than the earlier ones (Fig. 4d). We took BRMS1 as an example in Fig. 4c, which participates in the epidermal growth factor receptor (EGFR) and NF- $\mathrm{kB}$ signalling pathways [36]. The promoter methylation level of BRMS1 decreased, while its expression level increased across developmental stages.

\section{Regulatory networks of PCGs and non-coding RNAs}

We predicted the target genes for each miRNA and lncRNA, and further investigated whether the stagespecific PCGs at each developmental stage were highly enriched for targets of certain miRNAs and lncRNAs. If this was the case, then the enriched miRNAs and lncRNAs might be implicated in skin development. The detailed summary statistics are listed in Additional file 12: Table S6. For instance, TCONS_00394738, TCONS_ 00439958, and TCONS_00097544 were the top IncRNAs, whose targets were significantly $(\mathrm{FDR}<0.05)$ enriched for PCGs with specific expression at E85. The targets of these three lncRNAs were significantly (FDR < 0.05) engaged in immune response and the Notch signalling pathway (Fig. 5a). The targets of the top three significant $(F D R<0.05)$ miRNAs at E65, Oar-miR-150, oar-miR-200c, and oar-miR-152, were significantly (FDR $<0.05)$ enriched in epithelial development, immune response, and the Notch signalling pathway (Fig. 5b).
We further performed a competing endogenous RNA (ceRNA) analysis to detect the regulatory effects of circRNAs or lncRNAs on the expression of PCGs by mediating miRNAs during hair follicle morphogenesis. A detailed summary of the negative correlation (Pearson correlation coefficient $(\mathrm{PCC})<-0.7)$ of miRNA-target pairs is in Additional file 13: Table S7, while the positive correlation (PCC > 0.7) of ceRNA pairs (lncRNA-PCG and circRNA-PCG) which were targeted by a common miRNA (the hypergeometric test, $P<0.05$ ) is in Additional file 14: Table S8. We displayed the top 200 circRNA-miRNA-mRNA and the top 200 lncRNAmiRNA-mRNA interactions in Fig. 6a. The GO functional enrichment analysis revealed that genes in this ceRNA network were significantly enriched in skin development, keratinocyte proliferation, epidermal development (Fig. 6b). We proposed 12 most promising circRNAs and lncRNAs that acted as ceRNAs to affect the expression of stage-specific PCGs by sponging miRNAs during hair follicle morphogenesis (Fig. 6c). For instance, FGF7 exhibited the specific expression at E65 (Fig. 3e), which participates in skin development, keratinocyte proliferation, and cell proliferation. The expression of FGF7 was regulated by circRNA.18823, circRNA.688, and TCONS_00428946 through mediating oar-miR-200b and oar-miR-200c (Fig. 6c). Similarly, the expression of GRHL2 was regulated by TCONS 000493860 through mediating oar-miR-1197-3p, oarmiR-432, and oar-miR-494-3p. GRHL2 was specifically expressed at E85 and regulates epidermal cell

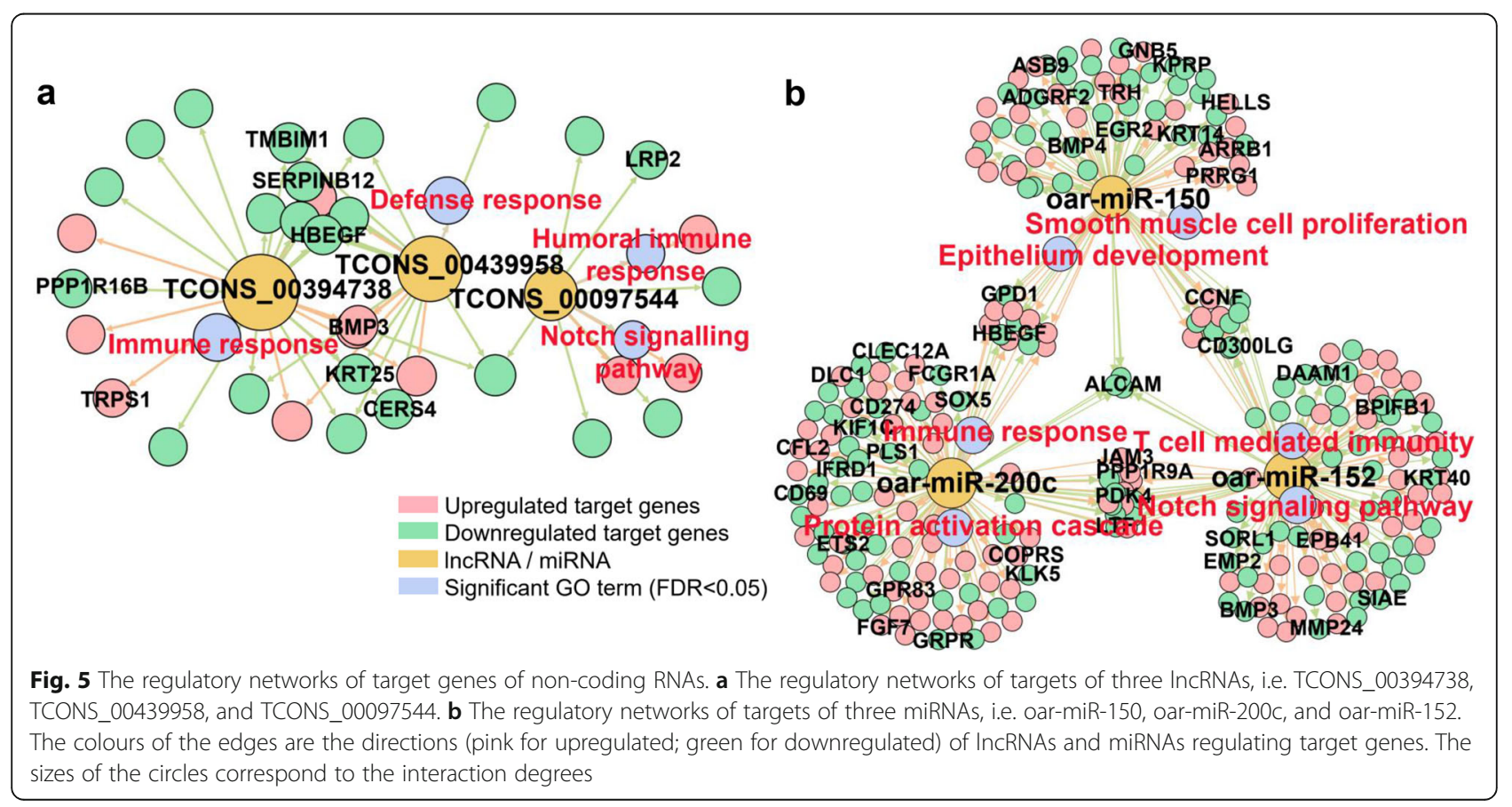




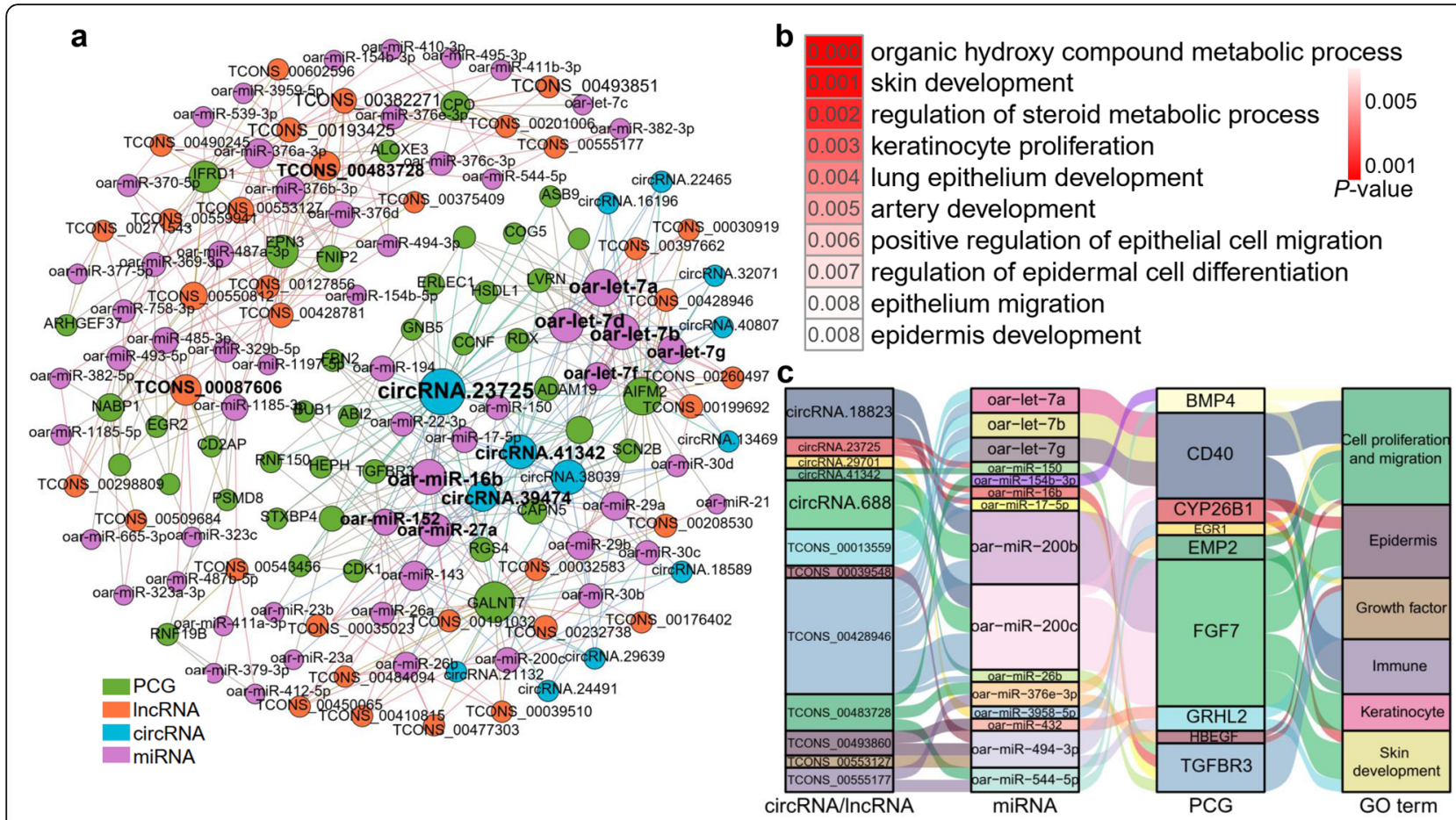

Fig. 6 Competing endogenous RNA (ceRNA) network. a The top 200 circRNA-miRNA-mRNA and top 200 IncRNA-miRNA-mRNA interactions. The edges are the Pearson correlation coefficient (PCC) between genes. The sizes of the circles correspond to the connection degrees. $\mathbf{b}$ Top10 enriched Gene Ontology (GO) terms (biological processes, BP) in ceRNAs comparing to the genome background. The $P$ values are computed by the hypergeometric test. c Sankey diagram of important candidate ceRNA pairs. Each rectangle represents a gene; degree of connection of each gene is directly proportional to rectangle size

differentiation and skin development [37] (Fig. 6c, Fig. 3e). Altogether, these results shed light on the complex interaction networks of PCGs and non-coding RNAs, which regulate hair follicle differentiation and growth.

\section{Gene co-expression network analysis}

We performed an unsigned weighted gene co-expression network analysis (WGCNA) among PCG, lncRNA, circRNA, and miRNA to identify co-expression modules related to hair follicle morphogenesis. In total, we detected 15 co-expression modules with the four gene types (Fig. 7a). The expression patterns of five modules were significantly (FDR <0.5) stage-specific (Fig. 7b). The GO functional enrichment analyses revealed that these stage-specific modules were significantly enriched in immune response, cell cycle phase, and metabolic process (Fig. 7c, Additional file 15: Fig. S7a). In addition, we found that many genes without functional annotation were co-expressed with functionally annotated genes (Additional file 15: Fig. S7 b, c). For instance, there were 1135 genes (347 PCGs, 635 lncRNAs, 153 circRNAs) with no functional information co-expressed with other annotated genes in a module (coloured brown), which were significantly enriched in the immunity system. These results indicated that our newly generated datasets can serve as a useful resource for functionally annotating genes in sheep.

We detected six TFs, whose motifs were significantly enriched in these five stage-specific modules (Fig. 7c). These TFs also showed stage-specific expression and participate in embryonic development. For instance, TFDP1 and E2F4 participate in the TGF- $\beta$ signalling pathway [38] and showed stage-specific expression at E65 and P7 (Fig. 7c). IRF1 serves as an activator of genes involved in the innate and acquired immune responses. It also enhanced the expression of interferon-kappa (IFк) [39] and showed stage-specific expression at E105 (Fig. 7c).

\section{Integrative analysis of stage-specific molecular features with GWAS signals of wool and growth traits}

To determine whether the developmental gene expression and regulation patterns allow us to better interpret the genetic variants associated with complex traits, we integrated the stage-specific molecular features detected above with GWAS signals of five wool traits and live weight in Merino sheep (Additional file 16: Table S9) [40]. As shown in Fig. 8a, b, genes with stage-specific expression were significantly (FDR $<0.1$ ) enriched for GWAS signals of all these traits. For instance, genes with 


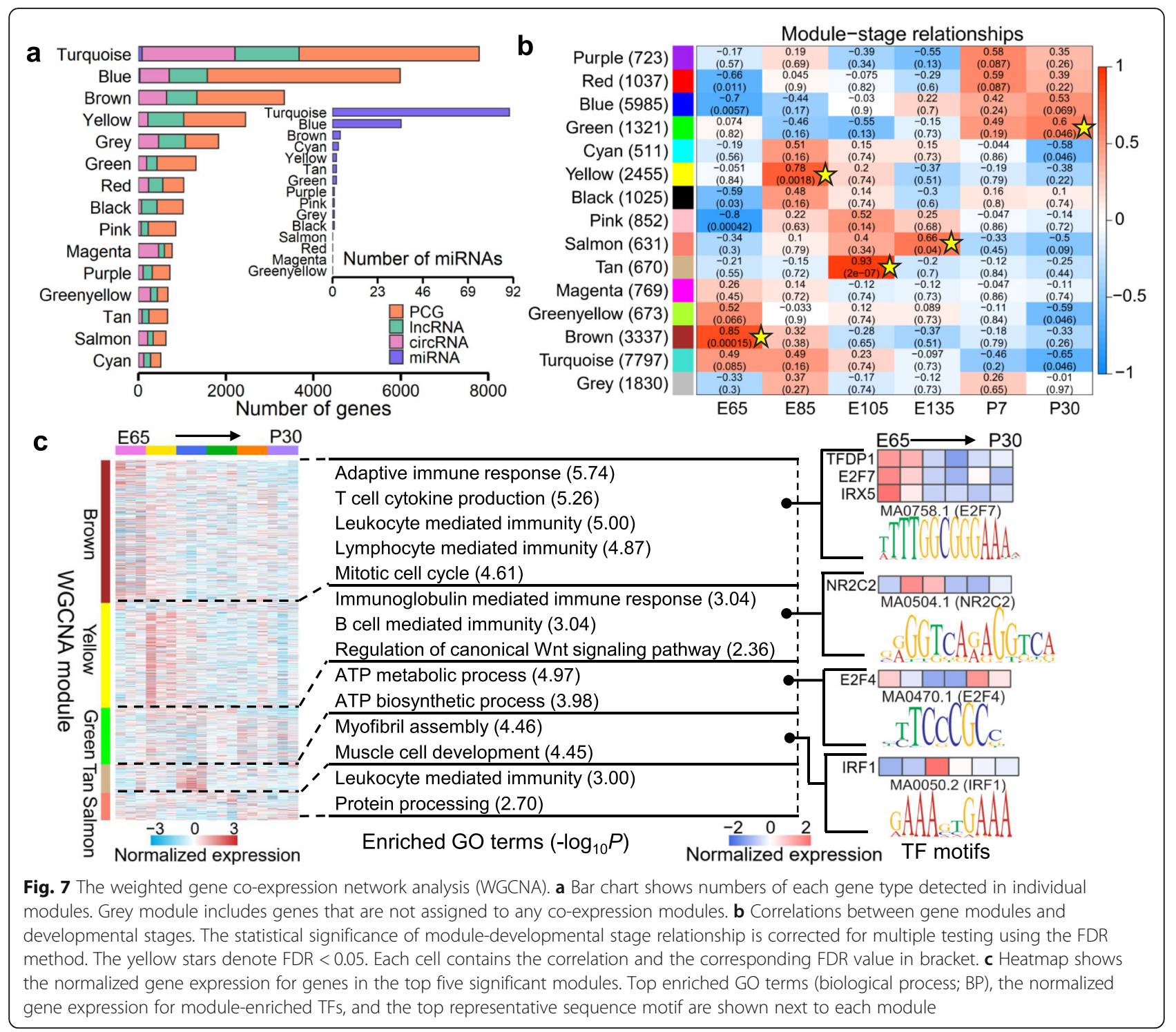

specific upregulation at E105 were significantly (FDR < 0.1 ) enriched in GWAS signals of five traits, including MFD, CVFD, CN, MSL, and LW (Fig. 8a). In addition, targets of miRNAs and circRNAs, which showed specific upregulation at E135 and P7, were significantly (FDR < 0.1 ) enriched for GWAS signals of CVFD. Targets of IncRNAs and miRNAs with P30 specific upregulation were significantly (FDR < 0.1) enriched in GFW (Fig. 8a). Furthermore, gene co-expression modules were also significantly enriched for GWAS signals of all six traits (Fig. 8c). For instance, the gene co-expression module coloured yellow, which was significantly enriched in immune responses and the regulation of canonical Wnt signalling pathway (Fig. 7c), was significantly (FDR < 0.1 ) enriched for GWAS signals of MFD, CVFD, and CN (Fig. 8c).
By comprehensively integrating GWAS [40], the transcriptome data from this study, the sheep expression atlas [41, 42], and the human GWAS atlas [43, 44], we proposed the most promising candidate genes for each of the six traits (Additional file 17: Table S10). For instance, the top SNP of SPHK1 explained $0.44 \%$ of the genetic variance (the fourth QTL region regarding the explained genetic variance) in CVFD and its expression level gradually increased during hair follicle development. SPHK1 was specifically and highly expressed in the immune system and was strongly associated with immune-related traits such as the reticulocyte fraction of red blood cells in humans (Fig. 8d). The top SNP of GHR explained $0.76 \%$ (the third QTL region) and $0.39 \%$ (the ninth QTL region) of the genetic variance in $\mathrm{CN}$ and MSL, respectively. GHR was gradually 


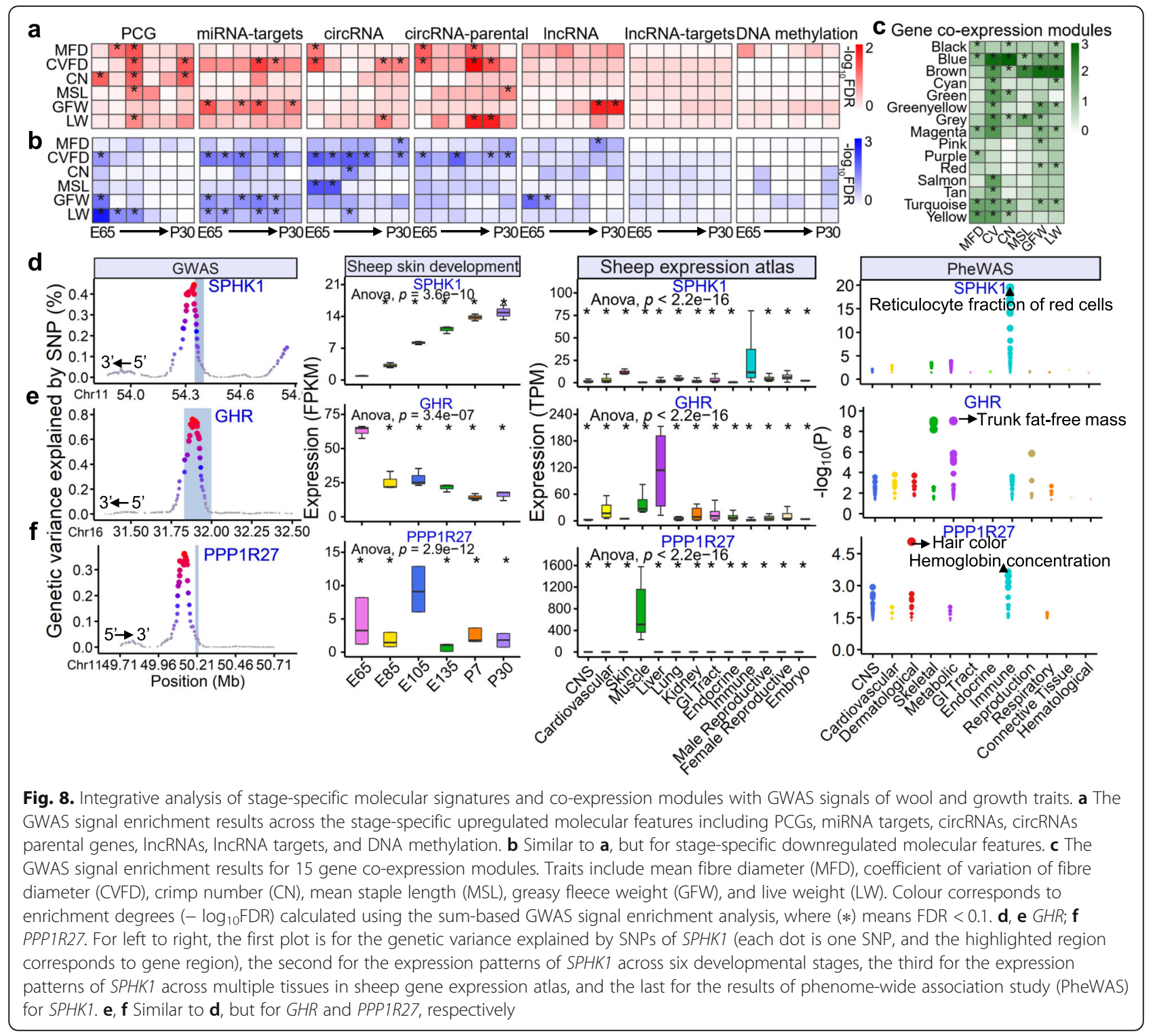

downregulated during hair follicle development. It was specifically expressed in liver and was significantly associated (FDR < 0.05) with metabolism-related traits such as trunk fat-free mass in humans (Fig. 8e). PPP1R27 showed the highest expression level at E105 compared to other stages, and its top SNP explained 0.36\%, 0.37\%, $0.24 \%$, and $0.22 \%$ of genetic variance in LW, MFD, GFW, and CV, respectively. It was specifically expressed in muscle and was significantly associated with hair colour and haemoglobin levels in humans (Fig. 8f). The explained genetic variance, expression patterns across tissues, and phenome-wide association study (PheWAS) results of CSRP2 for MFD, EEF1A2 for MSL, and PTPN1 for GFW are shown in Additional file 18: Fig. S8. In summary, the candidate genes discovered here showed specificity for developmental stage and tissue type, and their orthologues were associated with similar complex traits in humans.

\section{Discussion}

The elucidation of the morphology and molecular mechanisms underlying the normal development of sheep hair follicles expands our understanding of hair growth biology and the genetic basis of wool traits. In this study, we first explored the morphogenesis of hair follicles using the H\&E staining approach across six developmental stages, and demonstrated the asynchronous development of sheep hair follicles. Sheep and mouse exhibit the similar anatomical structure of the primary hair follicles, including the de novo formation of wool placode, dermal condensation, and the thickening of epidermis and dermis $[4,45]$. However, sheep has secondary hair follicles, 
sweat glands, and postnatal hair growth cycles, while mouse lacks them. This indicates that although mammals share similarity during hair follicle development, sheep might exhibit distinct morphology and regulation mechanisms [46].

We demonstrated that stage-specific PCGs, noncoding RNAs, and DNA methylation play a critical role in hair follicle development. We discovered several stage-specific TFs, such as KLF4, LEF1, HOXC13, RBPJ, VDR, RARA, and STAT3, associated with hair follicle development and growth [24-30]. Functional enrichment analysis indicated that stage-specific genes were significantly enriched in signalling, cell migration, and aggregation, highlighting the central roles of intercellular crosstalk and dynamic cell rearrangement in the hair morphogenesis. Specifically, it has been demonstrated that the hair follicle fate was regulated by the canonical Wnt/ $\beta$-catenin signalling [47], cellular differentiation by BMP signalling [48], and dermal papilla cell proliferation by Notch signalling [49].

We found that stage-specific genes (e.g. IFN, CD40 and TGFBR3) and co-expression modules were significantly enriched in the immune system. Previous studies proposed that hair follicles had an immune capacity in the growth stage of hair cycle, characterized by the downregulation of major histocompatibility complex (MHC) class I and the upregulation of potent immunosuppressants in mammals [50]. This hair follicle immune capacity is also regulated by pathways like NF-kappa B, CD antigens, interleukins (IL), TNFs, and IFNs related [51]. The collapse of this hair follicle immune capacity has been proposed to initiate the loss of hair as seen in patients with the autoimmune disease alopecia areata (AA) [52, 53].

According to the biological hypothesis of ceRNA [54], circRNAs and lncRNAs regulate the expression of target genes by modulating miRNAs $[55,56]$. In this study, by constructing ceRNA interaction networks using the stage-specific PCGs, miRNAs, circRNAs, and lncRNAs, we found that several circRNAs and lncRNAs regulated the expression of PCGs via modulating stage-specific miRNAs. We also found that a single miRNA can simultaneously target multiple circRNAs and lncRNAs, indicating that miRNA plays a central role in this interaction network by supplying multiple intermediate bridges linking circRNAs/lncRNAs to PCGs [57].

Identifying the causal genes and tissues for complex phenotypes contributes to animal breeding. Recent studies integrated various types of data from a wide range of tissues, such as gene expression $[17,58]$, DNA methylation [59], chromatin states [60, 61], histone modifications [62], and expression quantitative trait loci (eQTL) [63], with GWAS summary statistics to identify tissues and cell types that were relevant with complex traits and diseases. Here, by integrating developmental stagespecific PCGs, miRNAs, circRNAs, lncRNAs, and DNA methylation regions as well as gene co-expression modules with GWAS signals of wool and growth traits in sheep [40], we expanded our insights into the genetic architecture underlying such complex traits. However, functional experiments (e.g. gene editing) will be required to validate the candidate genes of wool traits identified in this study, and to assess their usefulness in animal breeding. In addition, the findings here can be further incorporated into genomic prediction models as biological priors, such as GFBLUP [64], for improving the prediction accuracy and for fine-mapping causal genes [17]. In future studies, additional omics data (e.g. ChIP-Seq, ATAC-Seq, Hi-C, and single-cell RNA-seq) from more tissue types (e.g. immune tissues) should be included to identify the molecular drivers underlying hair follicle development. Overall, the increasingly comprehensive functional annotations of genome will enable us to better elucidate the genetic basis of complex trait variation and to enhance the genetic improvement program in livestock [65].

\section{Conclusions}

In summary, we here characterized the global changes of the whole transcriptome and DNA methylation across six developmental stages of hair follicle in sheep. We identified the key molecular features that were significantly associated with hair follicle morphogenesis, and highlighted the complexity of the regulatory networks of PCGs and non-coding RNAs during the hair follicle development. Through integrating these findings with GWAS of wool traits, we provided novel insights into the genetic and biological mechanisms underpinning such traits in sheep. These datasets and findings provided a valuable resource for understanding the biology of hair follicle development and for interpreting the genetic basis of skin-relevant traits in mammals.

\section{Methods}

\section{Animal and tissue collection}

All the experimental animals were Subo Merino sheep obtained from Xinjiang Fine Wool Sheep Breeding Farm (Xinjiang, China). Subo Merino is a sub-type of Merino, which was bred in 2014 in China by crossing Australian Merino (paternal) with the Chinese Merino (maternal) [66]. Eighteen healthy Subo Merino ewes (2-3 years old; MFD, $18.1 \pm 0.5 \mu \mathrm{m}$ ) were artificially inseminated with fresh sperm from a Subo Merino ram (3 years old; MFD, $19.0 \pm 0.4 \mu \mathrm{m})$ and then managed at the same flock. The pregnant ewes were housed indoor for a 7-day "settlingin period" prior to being euthanized (electrocution followed by exsanguination). The embryos were collected in pregnant ewes at four embryonic days (i.e. E65, 
E85, E105, and E135). The skin tissues were collected immediately after euthanasia. The skin tissues of postnatal lambs were collected in vivo with approximately 2 $\mathrm{cm}^{2} \times 3 \mathrm{~mm}$ deep at P7 and P30. The wound was recovered in 2 weeks with care. Three biological replicates were generated for each of six developmental stages, and the body weight and length of all these 18 individuals were measured.

All eighteen skin tissues were collected from the right mid-side regions behind the shoulder blade of each individual and rinsed in $1 \times$ phosphate-buffered saline (PBS). Each skin tissue was divided into two parts: One was cut into a strip and fixed in $4 \%$ paraformaldehyde at $4{ }^{\circ} \mathrm{C}$ for $\sim 1$ week before H\&E staining. The H\&E staining was performed according to the classic method [67]. The skin samples were then made into paraffin sections. We selected three horizontal sections from each individual and 10 fields of view for each section, to count the average number of PF and SF by taking pictures with the same magnification using the electron microscope. The remaining skin samples were minced and snap-frozen in liquid nitrogen for the subsequent RNA extraction.

\section{Library construction and sequencing}

The total RNA from the 18 skin tissues was extracted using TRIzol reagent (Thermo Fisher Scientific, Waltham, MA, USA). All samples had high-quality RNA with RNA integrity number (RIN) > 8.0. For mRNA and lncRNA, the strand-specific sequencing libraries were constructed using the ribosomal RNA (rRNA) removal method following a previously described protocol [68]. For circRNA, the strand-specific sequencing libraries were constructed using rRNA-depleted and RNase Rdigested methods according to the previous protocol [69]. For miRNAs, the RNA molecules in a size range of 18-30 nt were enriched from total RNA by the polyacrylamide gel electrophoresis (PAGE). The 3' adaptors were then added, followed by the enrichment of RNAs with length of 36-44 nt and the ligation of $5^{\prime}$ adaptors to the RNAs. The ligation products were reversetranscribed by PCR amplification. All of the above libraries were sequenced on the Illumina NovaSeq6000 platform, which generated 150-bp paired-end (PE150) reads.

For MeDIP-Seq, the DNA libraries were prepared with a TruSeq DNA ample preparation kit (Illumina, San Diego, CA, USA). In brief, genomic DNA was extracted with a DNeasy blood \& tissue kit (Qiagen, Hilden, Germany). DNA $(3 \mu \mathrm{g})$ was sonicated in the range of $100-500$ bp. Subsequently, DNA underwent the endrepair, the generation of 3 '-dA overhangs, and adaptor ligation steps using a Paired-End DNA Sample Prep kit (Illumina, San Diego, CA, USA). DNA was then recovered by AMPure XP Beads and used for MeDIP using the Magnetic Methylated DNA Immunoprecipitation Kit
(Diagenode, Denville, NJ, USA) following the manufacturer's protocol. Adaptor-mediated PCR was performed to amplify the enriched fragments, and the library was sequenced on an Illumina HiSeq2500 PE150 platform.

\section{Pre-processing sequence data}

All raw reads were quality-tested with FastQC v. 0.11.8 (http://www.bioinformatics.babraham.ac.uk/projects/ fastqc/). For the mRNAs and lncRNAs, clean reads were obtained by removing adaptor and low-quality reads with Seqtk [70]. The rRNA-free reads were mapped to the sheep reference genome (Ensembl Oar_v3.1) using Hisat2 v. 2.4 [71]. Stringtie v. 1.3.1 [72, 73] was used to count the fragment within each gene. The expression of PCGs and lncRNAs was normalized as fragments per kilobase of exon model per million mapped fragments (FPKM). To compare the newly built Oar_rambouillet v1.0 with Oar_v3.1, we also mapped all 18 RNA-seq datasets to Oar_rambouillet_v1.0 using the same pipeline and found similar results in terms of mapping rates, gene expression, and stage-specific expression (Additional file 19: Table S11 and Additional file 20: Fig. S9). LncRNAs were defined as novel transcripts using the following filters: length $\geq 200 \mathrm{bp}$; number of exons $\geq 2$; ORF $\leq 300 \mathrm{bp}$; have no or weak protein-coding ability (CPC score $<0$ [74] \& CNCI score $<0$ [75] and no significant similarity with Pfam database [76]). Gffcompare v.0.9.8 [77] was used to compare lncRNAs derived from the current RNA-seq datasets with the known lncRNAs in NONCODE v5. The genes transcribed within $10 \mathrm{kbp}$ up- and downstream of an IncRNA were considered as its cis-acting target genes. The trans-acting target genes of lncRNAs were predicted using RNAplex software [9].

For the circRNAs, clean reads were obtained by fastp v. 0.18.0 [78]. Reads were then aligned to the reference genome by Hisat 2 v. 2.4 [71], and those with full length mapped were discarded. Next, from the unmapped reads, we extracted 20-nt from both ends and aligned them independently to find unique anchor positions within spliced exons by Hisat2 v. 2.4 [71]. Anchors aligning in the reverse orientation (head-to-tail) indicated circRNA splicing. They were then subjected to CIRI [79] to identify the circRNAs. The expression of circRNAs was quantified using the number of spanning back-spliced junction reads and normalized as spliced reads per billion mapping (SRPBM) [80].

For the miRNAs, clean tags were obtained by FASTXToolkit v. 0.0.13 [81] and aligned with small RNAs in the GeneBank (Release 209.0) and Rfam (Release 11.0) [82] databases to identify and remove rRNA, scRNA, snoRNA, snRNA, and tRNA. All clean tags were aligned to the sheep reference genome (Ensembl Oar_v3.1). The tags mapped to exons or introns from mRNA degradation and repeat sequences were removed. All clean tags 
were then searched against the miRBase (Release 22.1) [20] database to identify known miRNAs. The expression of miRNAs was calculated and normalized to counts per million (CPM). The target genes of miRNAs were predicted using three approaches, including mireap v. 0.20 [83], miRanda v. 3.3a [84], and TargetScan v. 7.0 $[85,86]$, and those detected by all three approaches were chosen for downstream analysis.

For the MeDIP-Seq data, raw reads were first processed to filter out low-quality reads that containing more than 5 "N"s or over $50 \%$ of the sequence with lowquality value (Phred score $<5$ ) using FASTX-Toolkit v. 0.0.13 [81]. The clean reads were aligned to the sheep reference genome, allowing up to two mismatches, using Bowtie v. 0.12.8 [87]. Peak calling were conducted using MACS v. 2.1.1 [88]. Peaks detected in individual samples from the same developmental stage were merged using BEDTools [89]. Genomic features were annotated in the $\mathrm{R}$ package ChIPseeker [90]. Promoters were defined as $1500 \mathrm{bp}$ up and $500 \mathrm{bp}$ downstream from the TSS of each gene. To evaluate the enrichment of methylation peaks, the fold enrichment ratio was calculated as the MeDIP-Seq counts relative to expected background counts $\lambda_{\text {local }}[88]$.

\section{Identification and annotation of stage-specific molecular features}

Stage-specific PCGs (FDR <0.05), lncRNAs (FDR < $0.05)$, circRNAs $(P<0.01)$, and miRNAs $(P<0.01)$ were identified between one stage and others using the $R$ package edgeR [91]. Stage-specific MRs $(P<0.01)$ were identified using the $R$ package DiffBind [92]. Stagespecific PCGs and circRNAs were separately clustered with the $\mathrm{R} k$-means function where $k=6$ within the cluster package according to the Euclidean distance. All functional enrichment analyses were conducted for each stage-specific gene types using the $\mathrm{R}$ package clusterProfile [93]. The edge $R$ package in $R$ [48] was used to calculate the fold changes of PCGs in each stage compared to the other stages. These fold changes were used as input data in the GSEA. GSEA was performed to establish whether a set of genes in specific GO terms were significantly differed from the other stages using the $\mathrm{R}$ package GSVA [94, 95], together with the annotated gene sets C5 v. 7.1 downloaded from the MsigDB database [96]. Significantly enriched gene sets $(F D R<0.05)$ were then ranked by the consensus score [97]. The top five representative gene sets (FDR < 0.05) with the largest consensus scores were selected for each stage and visualized with the $R$ package pheatmap [98]. The sequence motif enrichment analysis of promoters of stage-specific PCGs was conducted by MEME v. 5.3.3 [99], based on the JASPAR (2020) core non-redundant vertebrate motifs from Tomtom [99, 100].

\section{Regulatory network construction}

A hypergeometric test was used to determine whether stage-specific PCGs were significantly (FDR <0.05) enriched in miRNA or lncRNA targets. The regulatory networks of target genes of top three significantly (FDR $<0.05$ ) enriched lncRNAs and miRNAs were visualized using Gephi v. 0.9.2 [101].

The ceRNA network was constructed according to the following steps: (1) All stage-specific transcripts were selected and the expression correlation between PCGmiRNA or lncRNA-miRNA or circRNA-miRNA was evaluated using the PCC. Pairs with $\mathrm{PCC}<-0.7$ were selected as negatively co-expressed pairs. (2) Among all lncRNA-PCG and circRNA-PCG pairs, those with PCC $>0.7$ were selected as positively co-expressed pairs. (3) The hypergeometric test was used to determine whether the common miRNA sponges between the two genes were significant. Pairs with $P<0.05$ were selected. (4) The ceRNA network was constructed by assembling all co-expressed competing triplets, which were identified in (3), and visualized using Gephi v. 0.9.2 [101].

\section{Weighted gene co-expression network analysis (WGCNA)} An unsigned gene co-expression network was constructed using the $\mathrm{R}$ package WGCNA v. 1.12.0 [62]. Briefly, 29,616 PCGs and non-coding RNAs were used for the analyses. They all had expression >0.1 (FPKM for PCG and IncRNA, SRPBM for circRNA, CPM for miRNA) in $\geq 12$ samples. The normalized matrix was transformed to a matrix of Pearson correlations between gene pairs which, in turn, was converted to an adjacency matrix. To identify highly co-expressed gene modules, genes with similar expression patterns $(r>0.9)$ were clustered with a dynamic hybrid cutting algorithm. Eigengenes for these modules were defined as the first principal component of the corresponding expression matrix and were associated with all six hair follicle developmental stages.

\section{GWAS signal enrichment analysis}

The details of the weighted single-step genome-wide association study (WssGWAS) for five wool traits and one growth trait including MFD, CVFD, CN, MSL, GFW, and LW in Merino sheep was described previously [40]. Briefly, the GWAS population consisted of 7135 individuals (aged at 15 months) with phenotype data, among which 1217 had imputed high-density (HD) genotype data $(n=372,534)$ [40]. A sum-based marker-set test method was applied using the QGG package in $\mathrm{R}$ [17, 102] for GWAS signal enrichment analyses across stagespecific molecular features and gene co-expression modules: 


$$
T_{\text {sum }}=\sum_{i=1}^{m_{g}} b^{2}
$$

where $T_{\text {sum }}$ represents the summary statistics for each molecular feature, $\boldsymbol{m}_{\boldsymbol{g}}$ is the number of SNPs overlapping the molecular feature, and $\boldsymbol{b}$ is the SNP effect from GWAS. The marker-set sizes and LD patterns among markers were controlled by applying a genotype cyclical permutation strategy as described previously [103, 104]. To obtain empirical $P$ values for the association of a molecular feature with a complex trait, the permutation procedure was repeated 10,000 times and a one-tailed test was applied based on the proportion of random summary statistics greater than that observed $[17,102]$. The $P$ values were corrected for multiple testing using the FDR method. FDR $<0.1$ was considered significant.

\section{Detection of candidate genes of wool and growth traits with multiple data sources}

To detect candidate genes of wool and growth traits in sheep, we first focused on the stage-specific genes that were located in the top 50 ranked QTLs in terms of their explained genetic variance for each trait. The proportion of genetic variance explained by SNPs of candidate genes is derived from WssGWAS [40, 105]. To detect whether candidate genes show tissue-specific expression in a wide range of tissues and cell types, the gene expression estimates (transcripts per million, TPM) of 500 ovine samples were downloaded from the sheep gene atlas as reported by Clark et al. [41, 42]. They comprised 87 tissue and cell types with varying numbers of animals per tissue type. According to the known tissue biology [41], the samples were classified into 13 organ systems. The details are described in Additional file 21: Table S12.

The PheWAS has been widely used to associate a genetic variant with many phenotypes to explore its pleiotropic effect on complex traits [43]. To detect whether the human orthologues of candidate genes detected for wool and growth traits in sheep here are associated with similar traits in humans, a PheWAS analysis was conducted for each candidate gene based on the GWASAT LAS database [43, 44]. We used the GWAS summary statistics of 586 complex phenotypes from 159 publicly available human GWAS (the total sample size of each study $>2000$ ). We considered genes with adjusted $P$ values (FDR) less than 0.05 as significant. The complex traits were classified into 12 trait domains based on the known biology $[106,107]$. The details are described in Additional file 22: Table S13.

\section{Abbreviations}

CeRNA: Competing endogenous RNA; circRNA: Circular RNA; CN: Crimp number; CNS: Central nervous system; CPM: Counts per million; CVFD: Coefficient of variation of fibre diameter; FPKM: Fragments per kilobase of exon model per million mapped fragments; GFW: Greasy fleece weight; Gl Tract: Gastrointestinal tract; GO: Gene ontology; IncRNA: Long non-coding RNA; LW: Live weight; MeDIP-Seq: Methylated DNA immunoprecipitation sequencing; MFD: Mean fibre diameter; miRNA: MicroRNA; MRs: Methylated regions; MSL: Mean staple length; ncRNA: Non-coding RNA; PCA: Principal component analysis; PCC: Pearson correlation coefficient; PCG: Protein-coding gene; PheWAS: Phenome-wide association study; SRPBM: Spliced reads per billion mapping;

TF: Transcriptional factor; TSS: Ttranscriptional start site; WGCNA: Weighted gene co-expression network analysis; WssGWAS: Weighted single-step genomic association study

\section{Supplementary Information}

The online version contains supplementary material available at https://doi. org/10.1186/s12915-021-01127-9.

\begin{abstract}
Additional file 1: Fig. S1. The global study design. Grey boxes represent 18 samples collected from skin tissue at six developmental stages (three biological replicates per stage) of hair follicle in sheep. The $H \&$ Etaining method is used to observe the morphology of each sample. Orange boxes are for data generation, including strand-specific RNA-Seq for mRNA, InCRNA, circRNA and miRNA, as well as MeDIP-seq for DNA methylation in each of 18 skin samples. Green boxes show the major bioinformatics and statistical analyses involved in this study for functional annotation of stage-specific molecular signatures. Blue boxes describe other resources used for detecting candidate genes of wool traits in sheep. Pink boxes outline the main objective of this study, which is to reveal the genetic and biological basis of hair follicle development in sheep.
\end{abstract}

Additional file 2: Table S1. Mapping summary of all sequence data in this study.

Additional file 3: Table S2. Comparison of expressed genes detected in this study with known genes in sheep reference genome.)

Additional file 4: Table S3. The distribution of DNA methylation peaks among different genomic features.

Additional file 5: Fig. S2. Principal component analysis (PCA) of samples based on the expression levels of all four gene types including protein-coding genes (PCGs), IncRNAs, circRNAs and miRNAs.

Additional file 6: Fig. S3. General characteristics of circRNAs in sheep skin. $a$, Genomic origin of circRNAs in sheep skin. b, Distribution of parental genes (hosting genes) encoding different numbers of circRNAs in sheep skin.

Additional file 7: Fig. S4. Identification of stage-specific molecular signatures during sheep hair follicle development. $a, b, c, d$, e, and $f$ are numbers of stage-specific PCGs (FDR $<0.05)$, IncRNAs (FDR $<0.05)$, cirCRNAs $(P<0.01)$, miRNAs $(P<0.01)$, methylation regions (MRs) $(P<0.01)$ and genes overlapped in MRs, respectively. Solid lines: upregulation; dashed lines: downregulation.

Additional file 8: Table. S4. Top five representative Gene Ontology (GO) terms for upregulated and downregulated genes at each developmental stage revealed by a gene set enrichment analysis (GSEA).

Additional file 9: Table S5. Motif enrichment analysis for promoters of upregulated and downregulated stage-specific genes.

Additional file 10: Fig. S5. Functional enrichment analysis of downregulated circRNAs during sheep skin development. Heatmap shows the normalized expression of top 10 downregulated stage-specific circRNAs at each developmental stage. Bubble plot shows the top five enriched Gene Ontology (GO) terms (biological process; BP) for parental genes of downregulated stage-specific circRNAs.

Additional file 11: Fig. S6. Functional enrichment analysis of up- and down-regulated IncRNAs and miRNAs during sheep hair follicle development. Heatmaps show the normalized expression of top 10 upregulated and downregulated IncRNAs ( $a, b)$ and miRNAs ( $c, d)$ at each developmental stage. Bubble plots show the top five enriched gene ontology (GO) terms (biological process; BP) for targets of upregulated and downregulated IncRNAs $(a, b)$ and miRNAs $(c, d)$.

Additional file 12: Table S6. Number of significantly (FDR $<0.05$ ) enriched IncRNAs and miRNAs. 
Additional file 13: Table S7. Number of the negatively correlated (Pearson correlation coefficient $(P C C)<-0.7)$ miRNA-targets pairs.

Additional file 14: Table S8. Number of the positively correlated (Pearson correlation coefficient $(P C C)>0.7$ ) endogenous RNA (ceRNA) pairs targeted by a common miRNA $(P<0.05)$.

Additional file 15: Fig. S7. Gene co-expression modules determined by a weighted gene co-expression network analysis (WGCNA). a, Heatmap on the left shows the normalized gene expression of 15 modules. Top enriched Gene Ontology (GO) terms for each module are summarized on the right. b, Bar chart shows the numbers of annotated and unannotated genes in each module. c, Density chart shows the percentage of annotated and unannotated genes in each module. $\mathrm{b}$ and $\mathrm{c}$ share the same figure legend.

Additional file 16: Table S9. Significant GWAS signal enrichment results in wool and growth traits.

Additional file 17: Table S10. Summary of candidate genes for wool traits and live weight in Merino sheep.

Additional file 18: Fig. S8. Integrative analysis with multi-databases to detect candidate genes for wool traits and live weight in Merino sheep. a, CSRP2; b, EEF1A2; c, PTPN1. Plots (from left to right) show the genetic variance explained by SNPs of candidate gene (each dot is one SNP), the expression patterns of corresponding genes during sheep skin development, the expression patterns of corresponding genes across multitissues, and the phenome-wide association study (PheWAS) results for each candidate gene in humans, respectively.

Additional file 19: Table S11. Comparisons of the mapping statistics of 18 RNA-seq datasets between Oar_v3.1 and Oar_rambouillet_v1.0.

Additional file 20: Fig. S9. Comparison of gene expression for all 18 RNA-seq samples between Oar_v3.1 and Oar_rambouillet_v1.0. a. The Pearson correlation coefficient (PCC) of mean $\log _{2}($ count +1$)$ is $0.95, P$ value $<2.2 \mathrm{e}-16$; $b$. The PCC of mean $\log _{2}(\mathrm{FPKM}+1)$ is 0.94 , $P$-value $<2$.2e16. $c$ and $d$ The PCC of $\log _{2}($ count +1$)$ and $\log _{2}($ FPKM+1) for each sample, respectively. e and $\mathrm{f}$. Comparison of the stage-specific expression of PCGs between the two genome assemblies (Oar_v3.1 and Oar_rambouillet_v1.0).

Additional file 21: Table S12. Gene expression levels (transcripts per million, TPM) of six candidate genes in sheep gene expression atlas.

Additional file 22: Table S13. Phenome-wide association study (PheWAS) results for six candidate genes in humans.

\section{Acknowledgements}

We would like to thank Dr. Ablat Sulayman at the Xinjiang Academy of Animal Sciences and Dr. Yao Jiang at the National Engineering Laboratory for Animal Breeding of China Agricultural University for their helpful discussions and comments.

\section{Authors' contributions}

$\mathrm{KT}, \mathrm{SZ}$, and LF conceived and designed the study. BZ, JH, XH, and WZ collected the samples and/or generated the data. BZ, $\mathrm{HL}, \mathrm{SC}$, and $\mathrm{SL}$ conducted the statistical analyses. XF and YT managed the experimental sheep populations. BZ, LF, C-JL, and GEL drafted and revised the manuscript. All authors read and approved the final manuscript.

\section{Author's information}

Twitter handle: @LingzhaoF (Lingzhao Fang).

\section{Funding}

This work was supported by the National Natural Science Foundation of China (No. 31760655) and the China Agriculture Research System (Nos. CARS-36 and CARS-39). The funders played no role in study design, collection, analysis, data interpretation, manuscript writing, or decision to submit the manuscript for publication.

\section{Availability of data and materials}

All raw data generated in this study were submitted to the National Center for Biotechnology Information Sequence Read Archive (NCBI SRA) database under BioProject Nos. PRJNA705405 [108], PRJNA705547 [109], PRJNA705552
[110], and PRJNA705554 [111]. The GWAS summary data used in the current study were obtained from [40]. The sheep expression atlas was obtained from $[41,42]$. Human PheWAS data is available from $[43,44]$.

\section{Declarations}

Ethics approval and consent to participate

All animal procedures were conducted with the approval of the Animal Welfare Committee of China Agricultural University under Protocol No. DK996. All efforts were made to minimize animal suffering and discomfort.

\section{Consent for publication}

Not applicable.

\section{Competing interests}

The authors declare that they have no competing interests.

\section{Author details}

${ }^{1}$ National Engineering Laboratory for Animal Breeding, Key Laboratory of Animal Genetics, Breeding, and Reproduction, Ministry of Agriculture, College of Animal Science and Technology, China Agricultural University, Beijing, China. ${ }^{2}$ Key Laboratory of Genetics Breeding and Reproduction of the Fine Wool Sheep \& Cashmere Goat in Xinjiang, Institute of Animal Science, Xinjiang Academy of Animal Sciences, Urumqi, China. ${ }^{3}$ College of Animal Science, Xinjiang Agricultural University, Urumqi, China. ${ }^{4}$ Animal Genomics and Improvement Laboratory, Henry A. Wallace Beltsville Agricultural Research Center, Agricultural Research Service, Agricultural Research Service, USDA, Beltsville, Maryland, USA. ${ }^{5}$ MRC Human Genetics Unit at the Institute of Genetics and Cancer, University of Edinburgh, Edinburgh, UK. Institute of Animal Science and Veterinary Medicine, Shandong Academy of Agricultural Sciences, Jinan, China.

Received: 14 April 2021 Accepted: 18 August 2021

Published online: 09 September 2021

\section{References}

1. Schneider MR, Schmidt-Ullrich R, Paus R. The hair follicle as a dynamic miniorgan. Curr Biol Cb. 2009;19:132-42.

2. Fuchs E. Epithelial skin biology: three decades of developmental biology, a hundred questions answered and a thousand newones to address. Curr Top Dev Biol. 2016;116:357-74.

3. Rogers GE. Biology of the wool follicle: an excursion into a unique tissue interaction system waiting to be re-discovered. Exp Dermatol. 2006;15(12): 931-49. https://doi.org/10.1111/j.1600-0625.2006.00512.x

4. Nie Y, Li S, Zheng X, Chen W, Li X, Liu Z, et al. Transcriptome reveals long non-coding RNAs and mRNAs involved in primary wool follicle induction in carpet sheep fetal skin. Front Physiol. 2018:9:446. https://doi.org/10.3389/ fphys.2018.00446.

5. Streilein JW. Immune privilege as the result of local tissue barriers and immunosuppressive microenvironments. Curr Opin Immunol. 1993;5(3):42832. https://doi.org/10.1016/0952-7915(93)90064-Y.

6. Paus R, Nickoloff BJ, Ito T. A 'hairy' privilege. Trends Immunol. 2005;26(1):3240. https://doi.org/10.1016/j.it.2004.09.014.

7. Gibson WT, Westgate GE, Craggs Rl. Immunology of the Hair Follicle. Ann N Y Acad Sci. 2010;642:291-300.

8. Bertolini M, McElwee K, Gilhar A, Bulfone-Paus S, Paus R. Hair follicle immune privilege and its collapse in alopecia areata. Exp Dermatol. 2020; 29(8):703-25. https://doi.org/10.1111/exd.14155.

9. Saga K. Structure and function of human sweat glands studied with histochemistry and cytochemistry. Prog Histochem Cytochem. 2002;37(4): 323-86. https://doi.org/10.1016/S0079-6336(02)80005-5.

10. Wang $S, L i F$, Liu J, Zhang Y, Zheng Y, Ge W, et al. Integrative analysis of methylome and transcriptome reveals the regulatory mechanisms of hair follicle morphogenesis in cashmere goat. Cells. 2020;9(4):969. https://doi. org/10.3390/cells9040969.

11. Baubec T, Schuebeler D. Genomic patterns and context specific interpretation of DNA methylation. Curr Opin Genet Dev. 2014;25:85-92. https://doi.org/10.1016/j.gde.2013.11.015.

12. Zhao RR, Li J, Liu N, Li HG, Liu LR, Yang F, et al. Transcriptomic analysis reveals the involvement of IncRNA-miRNA-mRNA networks in hair follicle 
induction in Aohan fine wool sheep skin. Front Genet. 2020;11:590. https:// doi.org/10.3389/fgene.2020.00590.

13. Hindorff LA, Sethupathy P, Junkins HA, Ramos EM, Mehta JP, Collins FS, et al. Potential etiologic and functional implications of genome-wide association loci for human diseases and traits. Proc Natl Acad Sci U S A. 2009;106(23):9362-7. https://doi.org/10.1073/pnas.0903103106.

14. Andersson L, Archibald AL, Bottema CD, Brauning R, Burgess SC, Burt DW, et al. Coordinated international action to accelerate genome-to-phenome with FAANG, the functional annotation of animal genomes project. Genome Biol. 2015;16:57.

15. Liu S, Gao Y, Canela-Xandri O, Wang S, Yu Y, Cai W, et al. A comprehensive catalogue of regulatory variants in the cattletranscriptome. bioRxiv. 2020; https://doi.org/10.1101/2020.12.01.406280.

16. Ongen H, Brown AA, Delaneau O, Panousis NI, Nica AC, Dermitzakis ET, et al. Estimating the causal tissues for complex traits and diseases. Nat Genet. 2017;49(12):1676-83. https://doi.org/10.1038/ng.3981.

17. Fang LZ, Cai WT, Liu SL, Canela-Xandri O, Gao YH, Jiang JC, et al. Comprehensive analyses of 723 transcriptomes enhance genetic and biological interpretations for complex traits in cattle. Genome Res. 2020; 30(5):790-801. https://doi.org/10.1101/gr.250704.119.

18. Finucane HK, Reshef YA, Anttila V, Slowikowski K, Gusev A, Byrnes A, et al. Heritability enrichment of specifically expressed genes identifies diseaserelevant tissues and cell types. Nat Genet. 2018;50(4):621-9. https://doi.org/1 0.1038/s41588-018-0081-4.

19. Hormozdiari F, Gazal S, van de Geijn B, Finucane HK, Ju CJT, Loh PR, et al. Leveraging molecular quantitative trait loci to understand the genetic architecture of diseases and complex traits. Nat Genet. 2018;50(7):1041-7. https://doi.org/10.1038/s41588-018-0148-2.

20. Griffiths-Jones S, Saini HK, van Dongen S, Enright AJ. miRBase: tools for microRNA genomics. Nucleic Acids Res. 2008:36:D154-D58.

21. Wu J, Huang B, Chen H, Yin Q, Liu Y, Xiang Y, et al. The landscape of accessible chromatin in mammalian preimplantation embryos. Nature. 2016; 534(7609):652-7. https://doi.org/10.1038/nature18606.

22. Dussmann P, Pagel Jl, Vogel S, Magnusson T, Zimmermann R, Wagner E, et al. Live in vivo imaging of Egr-1 promoter activity during neonatal development, liver regeneration and wound healing. BMC Dev Biol. 2011; 11(1):28. https://doi.org/10.1186/1471-213X-11-28.

23. Kwon $\mathrm{H}-\mathrm{J}$, Bhat $\mathrm{N}$, Sweet EM, Cornell RA, Riley BB. Identification of early requirements for preplacodal ectoderm and sensory organ development. PLoS Genet. 2010;6(9):e1001133. https://doi.org/10.1371/journal.pgen.1 001133.

24. Ahmed NS, Ghatak S, El Masry MS, Gnyawali SC, Roy S, Amer M, et al. Epidermal E-cadherin Dependent beta-catenin pathway is phytochemical inducible and accelerates anagen hair cycling. Mol Ther. 2017;25(11):250212. https://doi.org/10.1016/j.ymthe.2017.07.010.

25. Phan QM, Fine GM, Salz L, Herrera GG, Wildman B, Driskell IM, et al. Lef1 expression in fibroblasts maintains developmental potential in adult skin to regenerate wounds. Elife. 2020;9:e60066. https://doi.org/10.7554/eLife.60066.

26. Wang SH, Luo ZX, Zhang YL, Yuan D, Ge W, Wang X. The inconsistent regulation of $\mathrm{HOXC13}$ on different keratins and the regulation mechanism on HOXC13 in cashmere goat (Capra hircus). BMC Genomics. 2018;19(1):630. https://doi.org/10.1186/s1 2864-018-5011-4.

27. Turkoz M, Townsend RR, Kopan R. The Notch intracellular domain has an RBPj-independent role during mouse hair follicular development. J Invest Dermatol. 2016;136(6):1106-15. https://doi.org/10.1016/j.jid.2016.02.018.

28. Bikle D, Christakos S. New aspects of vitamin D metabolism and action addressing the skin as source and target. Nat Rev Endocrinol. 2020;16(4): 234-52. https://doi.org/10.1038/s41574-019-0312-5.

29. Sato H, Koide T, Masuya H, Wakana S, Sagai T, Umezawa A, et al. A new mutation Rim3 resembling Re-den is mapped close to retinoic acid receptor alpha (Rara) gene on mouse Chromosome 11. Mamm Genome. 1998;9(1): 20-5. https://doi.org/10.1007/s003359900673.

30. Kim SM, Kang J, Yoon HS, Choi YK, Go JS, Oh SK, et al. HNG, a humanin analogue, promotes hair growth by inhibiting anagen-to-catagen transition. Int J Mol Sci. 2020;21(12):4553. https://doi.org/10.3390/ijms21124553.

31. Yu JS, Huang T, Zhang Y, Mao XT, Huang LJ, Li YN, et al. Substrate-specific recognition of IKKs mediated by USP16 facilitates autoimmune inflammation. Sci Adv. 2021;7:eabc4009.

32. Han S, Zhu T, Ding S, Wen J, Lin Z, Lu G, et al. Early growth response genes 2 and 3 induced by AP-1 and NF-kappa B modulate TGF-beta 1 transcription in NK1.1(-) CD4(+) NKG2D(+) T cells. Cell Signal. 2020;76: 109800.

33. Batista MR, Diniz P, Torres A, Murta D, Lopes-da-Costa L, Silva E. Notch signaling in mouse blastocyst development and hatching. BMC Dev Biol. 2020;20(1):9. https://doi.org/10.1186/s12861-020-00216-2.

34. Cheng L, Zhou MY, Gu YJ, Chen L, Wang Y. ZEB1: New advances in fibrosis and cancer. Mol Cell Biochem. 2021;476(4):1643-50. https://doi.org/10.1007/ s11010-020-04036-7.

35. Dong XM, Antao OQ, Song WZ, Sanchez GM, Zembrzuski K, Koumpouras F, et al. Type 1 interferon-activated STAT4 regulation of follicular helper T celldependent cytokine and immunoglobulin production in lupus. Arthritis Rheumatol. 2021;73(3):478-89. https://doi.org/10.1002/art.41532.

36. Zimmermann RC, Welch DR. BRMS1: a multifunctional signaling molecule in metastasis. Cancer Metastasis Rev. 2020;39(3):755-68. https://doi.org/10.1 007/s10555-020-09871-0.

37. Chen W, Liu ZX, Oh JE, Shin KH, Kim RH, Jiang M, et al. Grainyhead-like 2 (GRHL2) inhibits keratinocyte differentiation through epigenetic mechanism. Cell Death Dis. 2012;3(12):e450. https://doi.org/10.1038/cddis.2012.190.

38. Bao ZY, Zhao BH, Hu SS, Yang NS, Liu M, Li JL, et al. Characterization and functional analysis of SMAD2 regulation in hair follicle cycle in Angora rabbits. Gene. 2021;770:145339. https://doi.org/10.1016/.gene.2020.145339.

39. Lulli D, Carbone ML, Pastore S. Epidermal growth factor receptor inhibitors trigger a type I interferon response in human skin. Oncotarget. 2016;7(30): 47777-93. https://doi.org/10.18632/oncotarget.10013.

40. Clark EL, Bush SJ, McCulloch MEB, Farquhar IL, Young R, Lefevre L, et al. A high resolution atlas of gene expression inthe domestic sheep (Ovis aries). figshare https://doi.org/10.1371/journal.pgen.1006997.s004. 2017.

41. Clark EL, Bush SJ, McCulloch MEB, Farquhar IL, Young R, Lefevre L, et al. A high resolution atlas of gene expression in the domestic sheep (Ovis aries). PLoS Genet. 2017;13(9):e1006997. https://doi.org/10.1371/journal.pgen.1 006997.

42. GWASATLAS. https://atlas.ctglab.nl/PheWAS. Accessed 28 Dec 2020.

43. Watanabe K, Stringer S, Frei O, Mirkov MU, de Leeuw C, Polderman TJC, et al. A global overview of pleiotropy and genetic architecture in complex traits. Nat Genet. 2019;51(9):1339-48. https://doi.org/10.1038/s41588-019-04 81-0.

44. GWASATLAS. https://atlas.ctglab.nl/PheWAS. Accessed 28 Dec. 2020.

45. Muller-Rover S, Handjiski B, van der Veen C, Eichmuller S, Foitzik K, McKay IA, et al. A comprehensive guide for the accurate classification of murine hair follicles in distinct hair cycle stages. J Invest Dermatol. 2001;117(1):3-15. https://doi.org/10.1046/j.0022-202x.2001.01377.x.

46. Schmidt-Ullrich R, Paus R. Molecular principles of hair follicle induction and morphogenesis. Bioessays. 2005;27(3):247-61. https://doi.org/10.1002/bies.2 0184.

47. Andl T, Reddy ST, Gaddapara T, Millar SE. WNT signals are required for the initiation of hair follicle development. Dev Cell. 2002;2(5):643-53. https://doi. org/10.1016/S1534-5807(02)00167-3.

48. Rishikaysh P, Dev K, Diaz D, Qureshi W, Filip S, Mokry J. Signaling involved in hair follicle morphogenesis and development. Int J Mol Sci. 2014;15(1): 1647-70. https://doi.org/10.3390/ijms15011647.

49. Zhang HH, Nan WX, Wang SY, Zhang TT, Si HZ, Wang DT, et al. Epidermal growth factor promotes proliferation of dermal papilla cells via Notch signaling pathway. Biochimie. 2016;127:10-8. https://doi.org/10.1016/j. biochi.2016.04.015.

50. Paus R, Ito N, Takigawa M, Ito T. The hair follicle and immune privilege. J Investig Dermatol Symp Proc. 2003;8:188-94.

51. Hill RP, Haycock JW, Jahoda CAB. Human hair follicle dermal cells and skin fibroblasts show differential activation of NF-kappa B in response to proinflammatory challenge. Exp Dermatol. 2012;21(2):158-60. https://doi.org/1 0.1111/j.1600-0625.2011.01401.x.

52. Paus $\mathrm{R}$, Ito $\mathrm{N}$, Takigawa $\mathrm{M}$, Ito $\mathrm{T}$. The hair follicle and immune privilege. The journal of investigative dermatology Symposium proceedings / the Society for Investigative Dermatology, Inc European Society for Dermatological Research. 2003;8:188.

53. Tobin DJ. Characterization of hair follicle antigens targeted by the anti-hair follicle immune response. J Investig Dermatol Symp Proc. 2003;8(2):176-81. https://doi.org/10.1046/j.1087-0024.2003.00805.x.

54. Thomson DW, Dinger ME. Endogenous microRNA sponges: evidence and controversy. Nat Rev Genet. 2016;17(5):272-83. https://doi.org/10.1038/nrg.2 016.20 . 
55. Ashwal-Fluss R, Meyer M, Pamudurti NR, Ivanov A, Bartok O, Hanan M, et al. CircRNA biogenesis competes with pre-mRNA splicing. Mol Cell. 2014;56(1): 55-66. https://doi.org/10.1016/j.molcel.2014.08.019.

56. Chen LL. The expanding regulatory mechanisms and cellular functions of circular RNAs. Nat Rev Mol Cell Biol. 2020;21(8):475-90. https://doi.org/10.1 038/s41580-020-0243-y.

57. Liang RB, Han B, Li Q, Yuan YW, Li JG, Sun DX. Using RNA sequencing to identify putative competing endogenous RNAs (ceRNAs) potentially regulating fat metabolism in bovine liver. Sci Rep. 2017;7(1):6396. https://doi. org/10.1038/s41598-017-06634-W.

58. Pers TH, Karjalainen JM, Chan Y, Westra HJ, Wood AR, Yang J, et al. Biological interpretation of genome-wide association studies using predicted gene functions. Nat Commun. 2015;6(1):5890. https://doi.org/10.1 038/ncomms6890.

59. Fang L, Zhou Y, Liu S, Jiang J, Bickhart DM, Null DJ, et al. Comparative analyses of sperm DNA methylomes among human, mouse and cattle provide insights into epigenomic evolution and complex traits. Epigenetics. 2019;14(3):260-76. https://doi.org/10.1080/15592294.2019.1582217.

60. Trynka G, Sandor C, Han B, Xu H, Stranger BE, Liu XS, et al. Chromatin marks identify critical cell types for fine mapping complex trait variants. Nat Genet. 2013;45(2):124-30. https://doi.org/10.1038/ng.2504.

61. GTExConsortium. The Genotype-Tissue Expression (GTEx) pilot analysis: multitissue gene regulation in humans.Science. 2015;348:648-60.

62. Fang LZ, Liu SL, Liu M, Kang XL, Lin SD, Li BJ, et al. Functional annotation of the cattle genome through systematic discovery and characterization of chromatin states and butyrate-induced variations. BMC Biol. 2019;17(1):68. https://doi.org/10.1186/s12915-019-0687-8.

63. GTExConsortium. The genotype-tissue expression (GTEx) pilot analysis: Multitissue gene regulation in humans. Science.348:648-60.

64. Fang L, Sahana G, Ma P, Su G, Yu Y, Zhang S, et al. Use of biological priors enhances understanding of genetic architecture and genomic prediction of complex traits within and between dairy cattle breeds. BMC Genomics. 2017;18(1):604. https://doi.org/10.1186/s12864-017-4004-z.

65. Cardoso-Moreira M, Halbert J, Valloton D, Velten B, Chen C, Shao Y, et al. Gene expression across mammalian organ development. Nature. 2019; 571(7766):505-9. https://doi.org/10.1038/s41586-019-1338-5.

66. Liu N, Tian K, Shi G, He J, Liu J, Di J, et al. Effects of different generations on wool traits of Subo merino nucleus herds during upgrading crossing stages. Chin J Anim Sci. 2015;51:6-10.

67. Manuela M, et al. Eur J Histochem. 2016;60:76

68. Seqtk. https://github.com//h3/seqtk. Accessed 5 Jan 2020

69. Jeck WR, Sharpless NE. Detecting and characterizing circular RNAs. Nat Biotechnol. 2014;32(5):453-61. https://doi.org/10.1038/nbt.2890.

70. Seqtk. https://github.com/lh3/seqtk. Accessed 5 Jan 2020

71. Kim D, Landmead B, Salzberg SL. HISAT: a fast spliced aligner with low memory requirements. Nat Methods. 2015;12(4):357-60. https://doi.org/10.1 038/nmeth.3317.

72. Pertea M, Pertea GM, Antonescu CM, Chang TC, Mendell JT, Salzberg SL. StringTie enables improved reconstruction of a transcriptome from RNA-seq reads. Nat Biotechnol. 2015;33(3):290-5. https://doi.org/10.1038/ nbt.3122.

73. Pertea M, Kim D, Pertea GM, Leek JT, Salzberg SL. Transcript-level expression analysis of RNA-seq experiments with HISAT, StringTie and Ballgown. Nat Protoc. 2016;11(9):1650-67. https://doi.org/10.1038/nprot.2016.095.

74. Kong L, Zhang Y, Ye ZQ, Liu XQ, Zhao SQ, Wei L, et al. CPC: assess the protein-coding potential of transcripts using sequence features and support vector machine. Nucleic Acids Res. 2007;35(suppl_2):W345-9. https://doi. org/10.1093/nar/gkm391.

75. Pertea G, Pertea M. GFF Utilities: GffRead and GffCompare. F1000Res. 2020;9: 304.

76. Sun L, Zhang ZH, Bailey TL, Perkins AC, Tallack MR, Xu Z, et al. Prediction of novel long non-coding RNAs based on RNA-Seq data of mouse Klf1 knockout study. BMC Bioinformatics. 2012;13(1):331. https://doi.org/10.11 86/1471-2105-13-331.

77. Pertea G, Pertea M. GFF Utilities: GffRead and GffCompare. F1000Res. 2020;9: ISCB Comm J-304.

78. Chen SF, Zhou YQ, Chen YR, Gu J. fastp: an ultra-fast all-in-one FASTQ preprocessor. Bioinformatics. 2018;34:884-90.

79. FASTX-Toolkit. http://hannonlab.cshl.edu/fastx_toolkit/index.html. Accessed 2 Feb 2020.
80. Li Y, Zheng Q, Bao C, Li S, Guo W, Zhao J, et al. Circular RNA is enriched and stable in exosomes: a promising biomarker for cancer diagnosis. Cell Res. 2015;25(8):981-4. https://doi.org/10.1038/cr.2015.82.

81. mireap: discover new microRNA genes from small RNA sequencing reads. https://github.com/liqb/mireap. Accessed 1 July 2020.

82. miRanda database. http://www.microrna.org/microrna/home.do. Accessed 1 July 2020.

83. mireap: discover new microRNA genes from small RNA sequencing reads. https://github.com/liqb/mireap. Accessed 1 Jul 2020.

84. miRanda database. http://www.microrna.org/microrna/home.do. Accessed 1 Jul 2020.

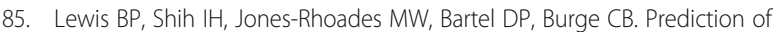
mammalian microRNA targets. Cell. 2003;115(7):787-98. https://doi.org/10.1 016/S0092-8674(03)01018-3.

86. Lewis BP, Burge CB, Bartel DP. Conserved seed pairing, often flanked by adenosines, indicates that thousands of human genes are microRNA targets. Cell. 2005;120(1):15-20. https://doi.org/10.1016/j.cell.2004.12.035.

87. Langmead B, Trapnell C, Pop M, Salzberg SL. Ultrafast and memory-efficient alignment of short DNA sequences to the human genome. Genome Biol. 2009;10(3):R25. https://doi.org/10.1186/gb-2009-10-3-r25.

88. Zhang Y, Liu T, Meyer CA, Eeckhoute J, Johnson DS, Bernstein BE, et al. Identifying ChIP-seq enrichment using MACS. Genome Biol. 2008;9(9):R137. https://doi.org/10.1186/gb-2008-9-9-r137.

89. Quinlan AR, Hall IM. BEDTools: a flexible suite of utilities for comparing genomic features. Bioinformatics. 2010;26(6):841-2. https://doi.org/10.1093/ bioinformatics/btq033.

90. DiffBind differential binding analysis of ChIP-Seq peak data. http:// bioconductor.org/packages/release/bioc/vignettes/DiffBind/inst/doc/ DiffBind.pdf. Accessed 20 July 2020.

91. Robinson MD, McCarthy DJ, Smyth GK. edgeR: a Bioconductor package for differential expression analysis of digital gene expression data. Bioinformatics. 2010;26(1):139-40. https://doi.org/10.1093/bioinformatics/ btp616.

92. DiffBind differential binding analysis of ChIP-Seq peak data. http:// bioconductor.org/packages/release/bioc/vignettes/DiffBind/inst/doc/ DiffBind.pdf. Accessed 20 July 2020.

93. Yu GC, Wang LG, Han YY, He QY. clusterProfiler: an R package for comparing biological themes among gene clusters. OMICS. 2012;16(5):2847. https://doi.org/10.1089/omi.2011.0118.

94. Molecular Signatures Database. http://www.gsea-msigdb.org/gsea/msigdb. Accessed 20 July 2020

95. Hanzelmann S, Castelo R, Guinney J. GSVA: gene set variation analysis for microarray and RNA-Seq data. BMC Bioinformatics. 2013;14(1):7. https://doi. org/10.1186/1471-2105-14-7.

96. pheatmap: Pretty Heatmaps. https://cran.r-project.org/web/packages/phea tmap/index.html. Accessed 30 Mar 2020.

97. Varemo L, Nielsen J, Nookaew I. Enriching the gene set analysis of genomewide data by incorporating directionality of gene expression and combining statistical hypotheses and methods. Nucleic Acids Res. 2013; 41(8):4378-91. https://doi.org/10.1093/nar/gkt111.

98. pheatmap: Pretty Heatmaps. https://cran.r-project.org/web/packages/phea tmap/index.html. Accessed 30 Mar 2020.

99. Amith MT, Fujimoto K, Tao C. NET-EXPO: A Gephi Plugin Towards Social Network Analysis of Network Exposure for Unipartite and Bipartite Graphs. HCl International 2019 - Posters : 21st international conference. 2019:1034:312.

100. Fornes O, Castro-Mondragon JA, Khan A, van der Lee R, Zhang X, Richmond PA, et al. JASPAR 2020: update of the open-access database of transcription factor binding profiles. Nucleic Acids Res. 2020;48(D1):D87-92. https://doi. org/10.1093/nar/gkz1001

101. Bastian M, Heymann S, Jacomy M. Gephi: an open source software for exploring and manipulating networks. In: Proceedings of the third international conference on weblogs and social media; 2009.

102. Rohde PD, Fourie Sorensen I, Sorensen P. qgg: an R package for large-scale quantitative genetic analyses. Bioinformatics. 2020;36(8):2614-5. https://doi. org/10.1093/bioinformatics/btz955

103. Rohde PD, Demontis D, Cuyabano BCD, Borglum AD, Sorensen P, Genomic Med Schizophrenia G. Covariance association test (CVAT) identifies genetic markers associated with schizophrenia in functionally associated biological processes. Genetics. 2016:203:1901-13. 
104. Sorensen IF, Edwards SM, Rohde PD, Sorensen P. Multiple trait covariance association test identifies gene ontology categories associated with chill coma recovery time in Drosophila melanogaster. Sci Rep. 2017;7(1):2413. https://doi.org/10.1038/s41598-017-02281-3.

105. Wang HY, Misztal I, Aguilar I, Legarra A, Fernando RL, Vitezica Z, et al. Genome-wide association mapping including phenotypes from relatives without genotypes in a single-step (ssGWAS) for 6-week body weight in broiler chickens. Front Genet. 2014;5:134.

106. Polderman TJC, Benyamin B, de Leeuw CA, Sullivan PF, van Bochoven A, Visscher PM, et al. Meta-analysis of the heritability of human traits based on fifty years of twin studies. Nat Genet. 2015;47(7):702-9. https://doi.org/10.1 038/ng.3285.

107. Goh Kl, Cusick ME, Valle D, Childs B, Barabási A-L. The human disease network. Proc Natl Acad Sci. 2007;104(21):8685-90. https://doi.org/10.1073/ pnas.0701361104

108. Zhao BR, Luo HP, He JM, Huang XX, Chen SQ, Fu XF, et al. Comprehensive analysis of miRNAs during Merino sheep hair follicle development. National Center for Biotechnology Information Sequence Read Archive (NCBI SRA) database, https://wwwncbinlmnihgov/bioproject/?term=PRJNA705552 (2021).

109. Zhao BR, Luo HP, He JM, Huang XX, Chen SQ, Fu XF, et al. Comprehensive analysis of long non-coding RNAs andmRNAs during Merino sheep hair follicle development. National Center for Biotechnology Information Sequence Read Archive(NCBI SRA) database, https://wwwncbinlmnihgov/ bioproject/?term=PRJNA705554 (2021).

110. Zhao BR, Luo HP, He JM, Huang XX, Chen SQ, Fu XF, et al. Comprehensive analysis of miRNAs during Merino sheep hair follicle development. SRA, https://wwwncbinlmnihgov/bioproject/?term = PRJNA705552. 2021.

111. Zhao BR, Luo HP, He JM, Huang XX, Chen SQ, Fu XF, et al. Comprehensive analysis of long non-coding RNAs and mRNAs during Merino sheep hair follicle development. SRA, https://wwwncbin/mnihgov/bioproject/?term = PRJNA705554. 2021.

\section{Publisher's Note}

Springer Nature remains neutral with regard to jurisdictional claims in published maps and institutional affiliations.

Ready to submit your research? Choose BMC and benefit from:

- fast, convenient online submission

- thorough peer review by experienced researchers in your field

- rapid publication on acceptance

- support for research data, including large and complex data types

- gold Open Access which fosters wider collaboration and increased citations

- maximum visibility for your research: over $100 \mathrm{M}$ website views per year

At $\mathrm{BMC}$, research is always in progress.

Learn more biomedcentral.com/submissions 